\title{
Unsupervised connectivity-based thresholding segmentation of midsagittal brain MR images
}

\author{
Chulhee Lee ${ }^{\mathrm{a}, *}$, Shin $\mathrm{Huh}^{\mathrm{a}}$, Terence A. Ketter ${ }^{\mathrm{b}}$, Michael Unser ${ }^{\mathrm{c}}$ \\ ${ }^{a}$ Division of Electrical Engineering, Yonsei University, 134 Shinchon-Dong, Seodaemoon-Gu, Seoul 120-749, South \\ Korea \\ ${ }^{\mathrm{b}}$ Department of Psychiatry and Behavioral Sciences, Stanford University School of Medicine, Stanford, \\ CA 94305-5723, USA \\ ${ }^{\mathrm{c}}$ IOA, Biomedical Imaging Group, EPFL, DMT/IOA, P.O. Box 127, 1015 Lausanne, Switzerland
}

Received 13 February 1997; received in revised form 10 June 1998

\begin{abstract}
In this paper, we propose an algorithm for automated segmentation of midsagittal brain MR images. First, we apply thresholding to obtain binary images. From the binary images, we locate some landmarks. Based on the landmarks and anatomical information, we preprocess the binary images, which substantially simplifies the subsequent operations. To separate regions what are incorrectly merged after this initial segmentation, a new connectivity-based threshold algorithm is proposed. Assuming that some prior information about the general shape and location of objects is available, the algorithm finds a boundary between two regions using the path connection algorithm and changing the threshold adaptively. In order to test the robustness of the proposed algorithm, we applied the algorithm to 120 midsagittal brain images and obtained satisfactory results. (C) 1998 Elsevier Science Ltd. All rights reserved.
\end{abstract}

Keywords: Segmentation; Thresholding; Connectivity; Magnetic resonance image (MRI); Path connection algorithm

\section{Introduction}

Magnetic resonance (MR) imaging produces sequences of two dimensional images of three dimensional objects. In particular, MR images of the brain have provided valuable information in understanding brain anatomy. Although the sequences of two dimensional images provide

\footnotetext{
* Corresponding author.
} 
basic information on structure, abnormality, etc., further processing on the sequences provides far more information. For example, 3-D reconstruction from the sequences of 2-D images provides 3-D information of brain structure and allows volumetric analysis and coregistration with functional brain images. In order to obtain a 3-D representation, one needs to separate the brain from the surrounding tissues. This procedure, known as stripping, can be viewed as a special application of image segmentation [11-18]. However, due to the complexity of the brain structure and a large variation among individuals, researchers have not yet been entirely successful in developing a completely automated segmentation algorithm for brain MR images. Since segmentation of brain MR images is important in many medical image analysis, it has also been studied by many authors $[1,2,19,20]$. Kennedy et al. proposed a segmentation algorithm based on contouring and outline optimization [3]. Joliot and Mazoyer proposed a 3D segmentation algorithm using thresholding and a connectivity algorithm [4]. Some researchers applied neural networks for segmentation of MR images [5,6]. However, none of these approaches has been fully automated.

Among the various approaches available in segmentation, thresholding is by far the simplest and still most widely used [7-10]. Unfortunately, it is not always applicable because often there is not a single threshold (or a combination of thresholds) that properly separates the various objects in an image, particularly in medical imaging. In this paper, in order to successfully segment midsagittal brain MR images, we address some of the deficiencies of global thresholding methods by making use of simple a priori knowledge that can be translated into connectivity constraints. We propose a post-processing method that refines a initial segmentation map by separating objects that have been improperly merged together. The basis of the method is to adaptively adjust the threshold to meet certain connectivity constraints.

In our segmentation algorithm of midsagittal brain MR images, we first apply thresholding to obtain binary images. From the binary images, we locate some landmarks. Based on the landmarks and anatomical information, we preprocess the binary images to eliminate small regions and remove the skull, which substantially simplifies the subsequent operations. To identify regions that have been incorrectly merged, we make use of general a priori information obtained from the landmarks. Finally, we apply the new connectivity-based thresholding segmentation to separate brain regions that are improperly merged.

\section{Thresholding and locating landmarks}

Fig. 1 shows an original gray scale brain MR image and Fig. 2 its binary version obtained by applying thresholding. Although the brain is easily recognizable in the binary image, the problem is that there is no single threshold which will correctly separate it from its neighboring structures. Generally, some parts of the brain tend to be improperly connected to nonbrain tissues. In this paper, we starts the segmentation with the binary image. We try to perform segmentation as much as possible in binary images, and then return to the original gray scale images to solve problematic regions which could not be determined in binary images.

Segmentation without prior knowledge seldom produces desirable results. Assuming prior knowledge about images will limit the application of an algorithm. However, the result will be 


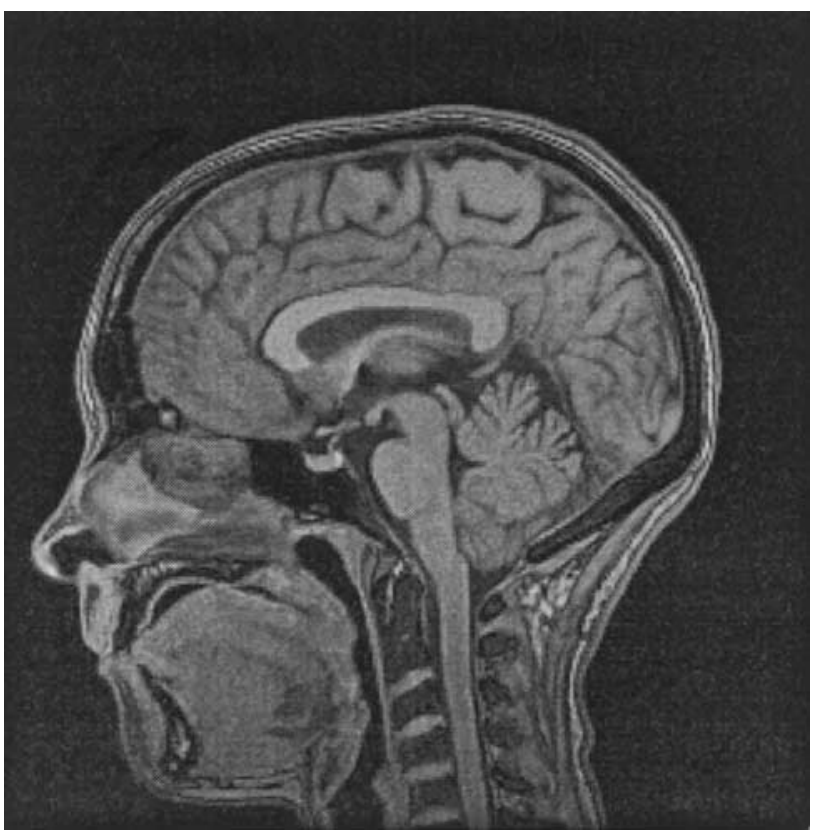

Fig. 1. Original gray scale brain MR image.

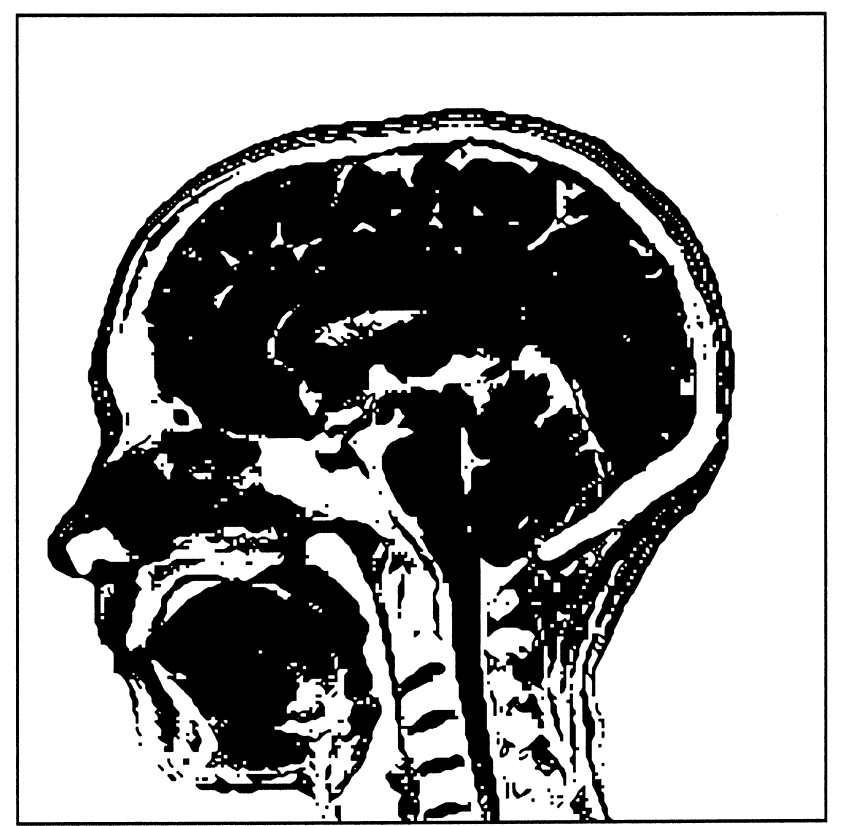

Fig. 2. Binary brain MR image. 
far more useful in many cases. In this paper, we utilize anatomical information to guide segmentation of the brain. This is achieved in part by locating some landmarks. If errors occur in locating the landmarks or the anatomical information is incorrectly applied, all subsequent operations will be unreliable. Therefore, we try to select landmarks which are robust and very unlikely to be incorrectly located and utilize anatomical information which is robust and subject-independent.

We use two landmarks which are easy to find and very unlikely to be erroneously located. First we locate the tip of the nose and then find the nasion (around the eye area) as shown in Fig. 3. Inside the skull, there is fluid between the skull and the brain and the fluid has very small values in MR images. Thus, we can assume that the fluid will have a background value in the binary image and that the forebrain (frontal lobes) is entirely above the nasion. This anatomical assumption is reasonable and can be applied to almost all subjects without any problem.

Next, preprocessing is performed on the binary images. First, we apply a connected component labeling and eliminate all regions but the largest one. Fig. 4 shows the MR brain image after this procedure. It can be seen that all small regions are removed and there remains only one connected region. Then, we eliminate small holes inside the brain and make three openings in the skull, one at the nasion, one posteriorly in the same horizontal plane and one near inferior and posterior limit of the brain as shown in Fig. 5. Finally, we apply the connected component algorithm to find regions and eliminate all regions except the largest one. This operation removes the skull. After this preprocessing, only one connected region remains without any hole. Fig. 6 shows the image resulting from preprocessing.

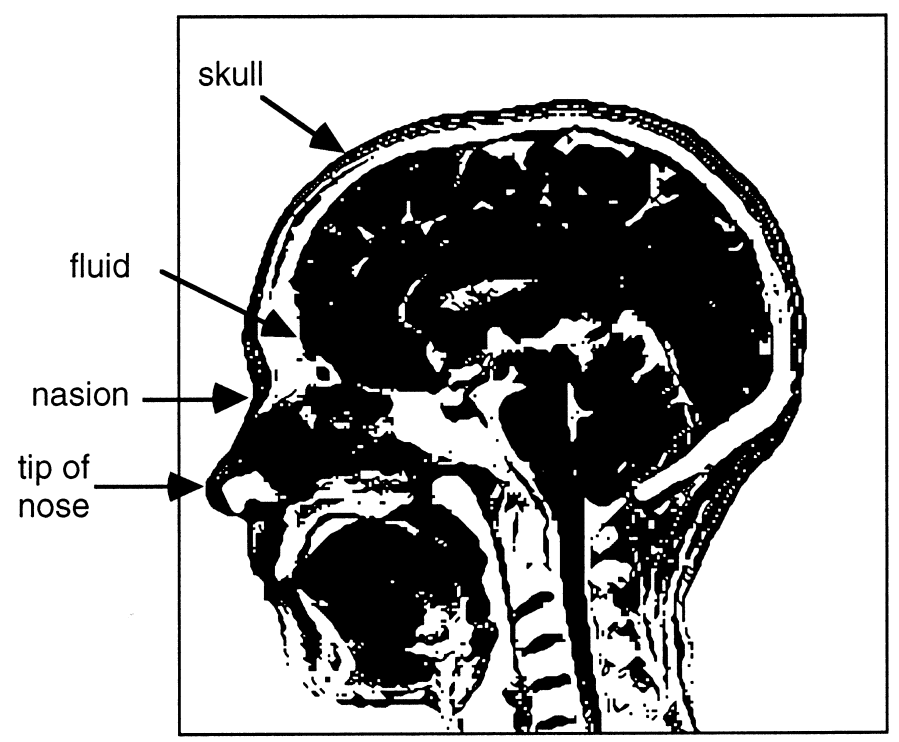

Fig. 3. Example of landmarks of human brain. 


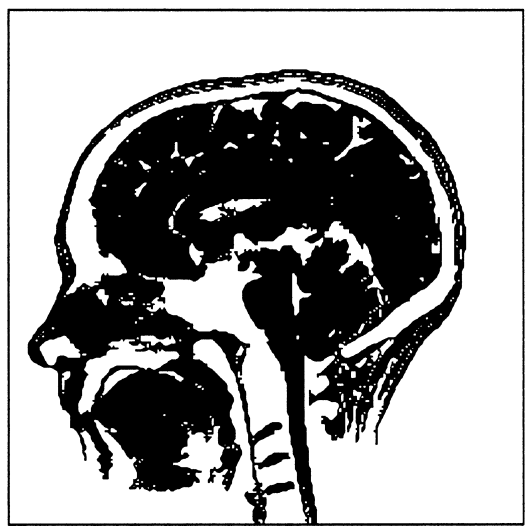

Fig. 4. Eliminating small regions.

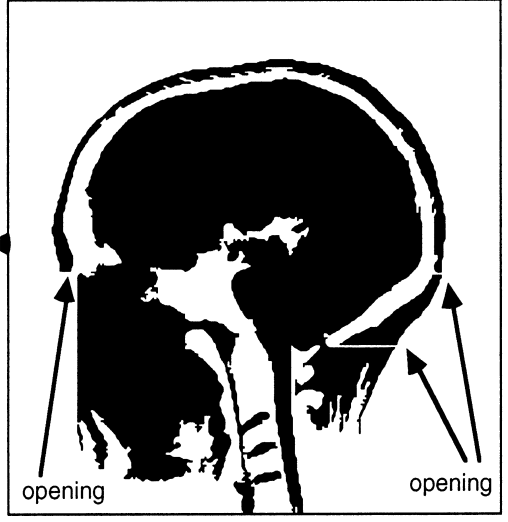

Fig. 5. Removing small holes and making openings in the skull.

\section{Connectivity-based threshold segmentation}

\subsection{Boundary and path connection algorithms}

One of the most difficult regions to process in sagittal brain MR images is the inferior aspect of the forebrain (inside the rectangular box in Fig. 7a) where, due to bone, eye muscles and other structures, the brain tends to be connected to surrounding nonbrain tissues. Fig. $7 \mathrm{c}$ shows a magnified view of this area. Usually, there is a boundary between the brain and adjacent structures. However, the width and the gray levels of this boundary can vary considerably. Global threshold methods ( $k$-means) or gradient methods typically fail in this kind of problem. This is exemplified in Fig. $7 \mathrm{~b}$ which shows the result of a global threshold method where the brain is merged together with other nonbrain tissues. Fig. $7 \mathrm{~d}$ displays the corresponding gradient image. Similar segmentation problems also occur in other medical images such as computer assisted tomography (CAT).

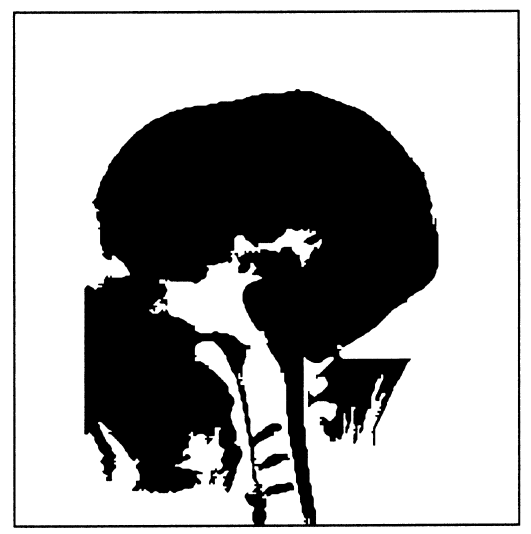

Fig. 6. Removing the skull. 
(a)

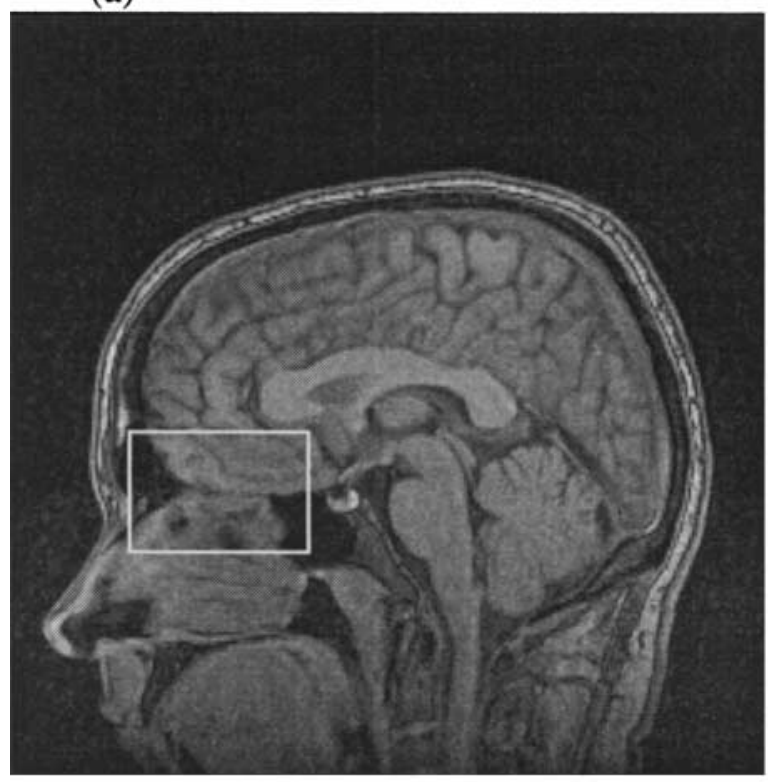

(c)

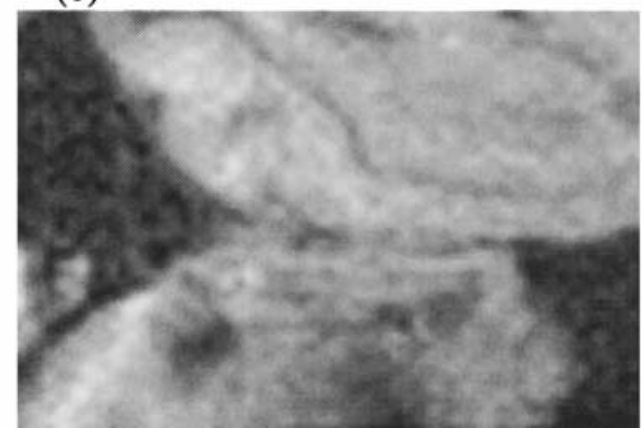

(b)

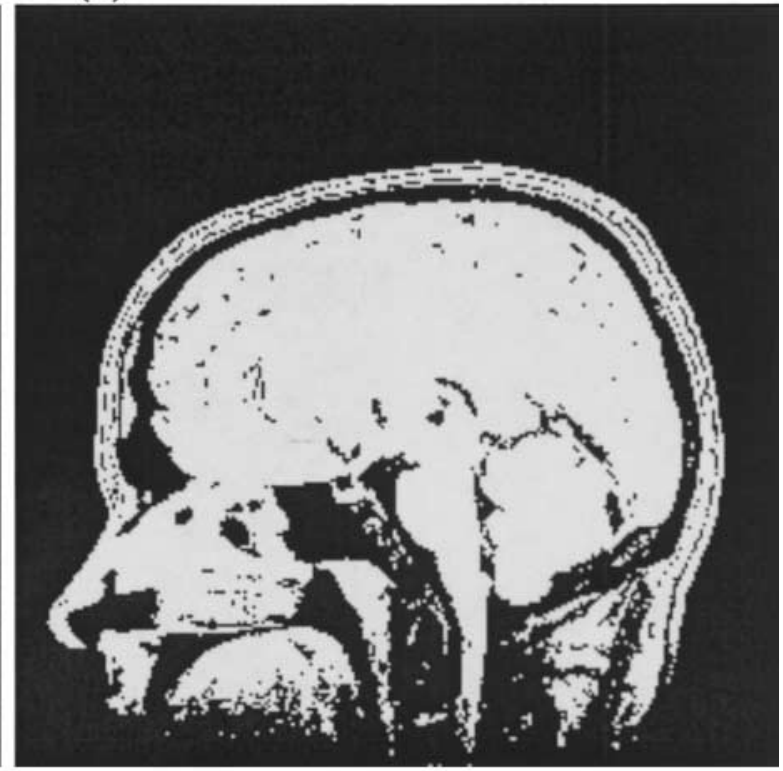

(d)

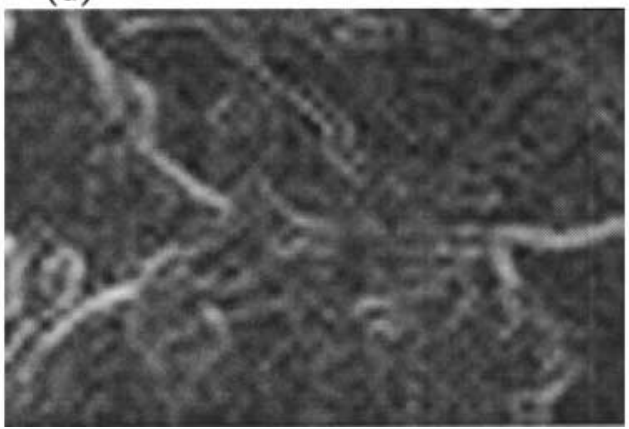

Fig. 7. Separating brain from surrounding tissues (a) original image (b) binary image (c) enlarged image (d) gradient image.

The problem with images of this nature is that the boundaries between regions are not always well defined. In this paper, we address segmentation in such a context and consider the segmentation task as finding the correct boundary between two regions. This simple definition can be applied to any image, although there can be a singular point where more than two regions meet, as shown in Fig. 8. However, for most practical purposes, such singular points may be ignored. There can be many criteria for distinguishing regions, such as gray level, texture and existence of a boundary. In practice, region characteristics may not be uniform and there can be significant variation within the same region; boundaries between regions may not be well defined. Here, we propose a new approach for finding such boundaries. The algorithm assumes that some prior information is available and that there exists a detectable boundary between two regions. The boundary may not be uniform and its location may be unknown. 


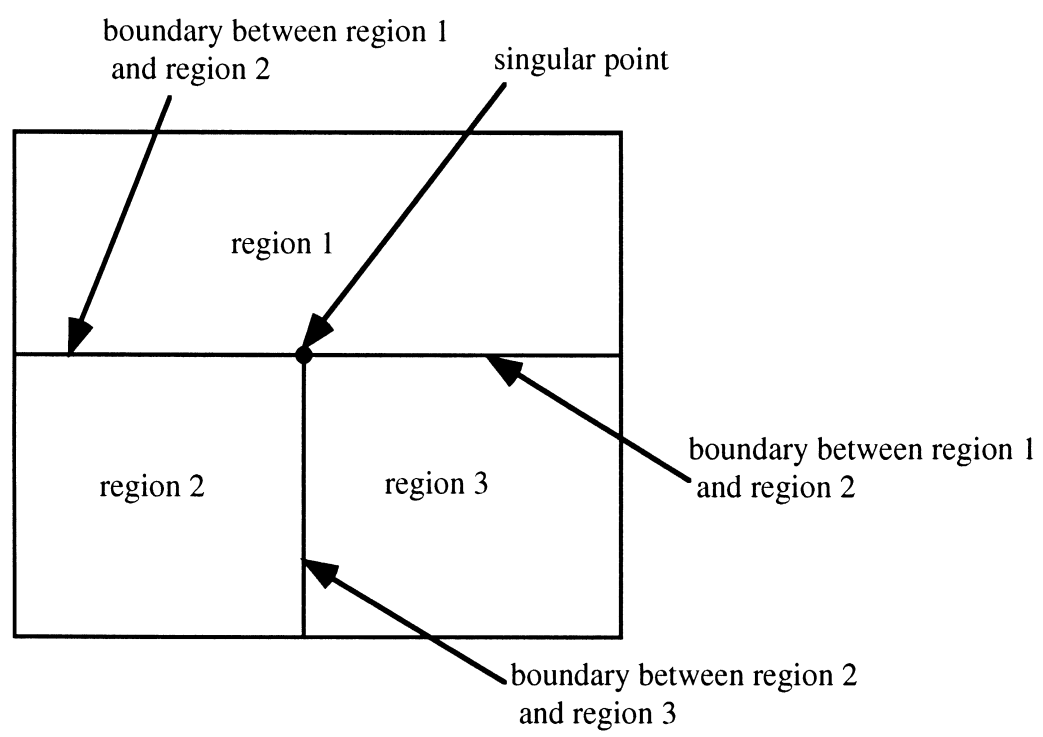

Fig. 8. Schematic of image segmentation.

Let us now briefly discuss the path connection algorithm that will be used in our segmentation method. The basic problem is to find a path between two points. One of the most widely used path connection algorithms is the Lee path algorithm [21] which always finds a path if any exists. There are also many variants of this algorithm to speed up the search procedure [22]. We propose to use this search technique to adaptively select a proper threshold that separates two objects. Specially, we will select two reference points and test whether these points are connected or not.

To be precise in describing our algorithm, we define a path function, $P_{\mathrm{v}}(\mathrm{a}, \mathrm{b})$, as follows:

$$
\begin{aligned}
& P_{\mathrm{v}}(\mathrm{a}, \mathrm{b})=1 \text { if there exists a path between } \mathrm{a} \text { and } \mathrm{b}, \\
& P_{\mathrm{v}}(\mathrm{a}, \mathrm{b})=0 \text { if there exists no path between a and } \mathrm{b},
\end{aligned}
$$

where $\mathrm{v}$ is the path value. It is noted that, in the definition of $P$, we include the path value. For example, in binary images, the path value can be either 0 or 1.

We will consider two cases. First, we assume that the end points of the boundary are known. In many practical situations, it is possible to find the intersection points when two objects are overlaid. Second, we consider the case in which one point in each object is known without any further knowledge of the location of the boundary.

\subsection{Separating two objects with two known end points}

Fig. 9 shows an illustration of the first case. There is a boundary which separates two objects and has two known end points $\mathrm{p}_{1}$ and $\mathrm{p}_{2}$. The boundary may or may not have some 


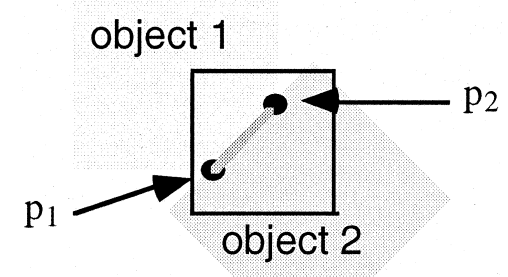

Fig. 9. Separating objects when two end points are given.

width. In other words, the boundary may consist of pixels which do not belong to adjacent regions, or be an imaginary line between the two different objects. The segmentation algorithm can be applied to both cases. Here we will consider the former case.

Without loss of generality, we can assume that the background value is zero and that the mean value of the boundary area is smaller than those of the objects. Now we draw a rectangular box containing the points $\mathrm{p}_{1}$ and $\mathrm{p}_{2}$ with some margin. Starting with $t=t_{0}$, we apply a threshold to the original gray scale image $\mathrm{X}$ inside the box to obtain a binary image $\mathrm{Y}$, i.e.

$$
y_{i j}=1 \text { if } x_{i j} \geq t, \quad y_{i j}=0 \text { otherwise. }
$$

Then we check if there exists a path between $\mathrm{p}_{1}$ and $\mathrm{p}_{2}$ with value 0 inside the box (i.e. objects 1 and 2 are disconnected). If there is one, i.e. $P_{0}\left(\mathrm{p}_{1}, \mathrm{p}_{2}\right)=1$ (see Eq. (1)), we separate the two objects. Otherwise, i.e. $P_{0}\left(\mathrm{p}_{1}, \mathrm{p}_{2}\right)=0$, the two objects are still connected. Then we increase the threshold and check again. Since we assumed that the mean value of the boundary area is smaller than those of the objects, the path will have zero values before the objects begin to be eroded. In addition, as the threshold increases, we will eventually find a path between the two points. In other words, we will always find a complete boundary, although the boundary may not be necessarily the true one. This property is a real advantage. The segmentation algorithm will never have problems of broken or missing boundaries. In the case in which the boundary does not have any area, i.e. the boundary is just an imaginary line between points of the objects, the same method can be used. We summarize the procedure as follows:

Procedure 1. Separating two objects (end points of the boundary are known):

Step 1: Initialize the threshold value $\left(t=t_{0}\right)$.

Step 2: Draw a rectangular box containing the two end points $\left(\mathrm{p}_{1}, \mathrm{p}_{2}\right)$ of the boundary with some margin.

Step 3: Apply the threshold $t$ to the pixels inside the box.

Step 4: Check if there exists a path inside the box between $\mathrm{p}_{1}$ and $\mathrm{p}_{2}$ with path value 0 . If there is one, i.e. $P_{0}\left(\mathrm{p}_{1}, \mathrm{p}_{2}\right)=1$, we separate the two objects. Otherwise, increase the threshold value and go to step 3. 


\subsection{Separating two objects with unknown boundary}

Now we consider the second case in which the location of the boundary is unknown. The situation is illustrated in Fig. 10. As previously, the boundary may or may not have some width. Here we do not make any assumption about the boundary.

We are given two points: $\mathrm{k}_{1}$ belonging to object 1 and $\mathrm{k}_{2}$ belonging to object 2 . Similarly we draw a box containing $\mathrm{k}_{1}$ and $\mathrm{k}_{2}$. Then we start with $t=t_{0}$, we apply a threshold to the image (Eq. (2)). Then we check if there exists a path between $\mathrm{k}_{1}$ and $\mathrm{k}_{2}$ with path value 1 . If there exists no path, i.e. $P_{1}\left(\mathrm{k}_{1}, \mathrm{k}_{2}\right)=0$ (see Eq. (1)), we separate the two objects. If there is one, i.e. $P_{1}\left(\mathrm{k}_{1}, \mathrm{k}_{2}\right)=1$, the two objects are still connected. Thus, we increase the threshold and check again. In practice, more than one point can be known from each object. In that case, we can repeat the procedure for several combinations of points to ensure that the results are consistent. We summarize the procedure as follows:

Procedure 2. Separating two objects (one point from each object is given):

Step 1: Initialize the threshold value $\left(t=t_{0}\right)$.

Step 2: Draw a rectangular box containing the two points $\left(\mathrm{k}_{1}, \mathrm{k}_{2}\right)$ with some margin.

Step 3: Apply the threshold to the pixels inside the box containing the two points with threshold value $t$ to the gray scale image $\mathrm{X}$ to obtain binary image $\mathrm{Y}$.

Step 4: Check if there exists a path between $\mathrm{p}_{1}$ and $\mathrm{p}_{2}$ with path value 1 . If there exists no path, we separate the two objects. If there is one, increase the threshold and go to step 3.

The scheme can be easily extended for texture segmentation provided that we can define a invariant discriminative function $F$. An example of such a function is given in [23]. The discriminative function $\mathrm{F}$ is designed to produce an output image $\mathrm{Y}$ from the input image $\mathrm{X}$ in such a way that the distributions of the two regions are separable in Y. In other words, after feature extraction, the distribution of the two regions are bimodal and separable. For the cases

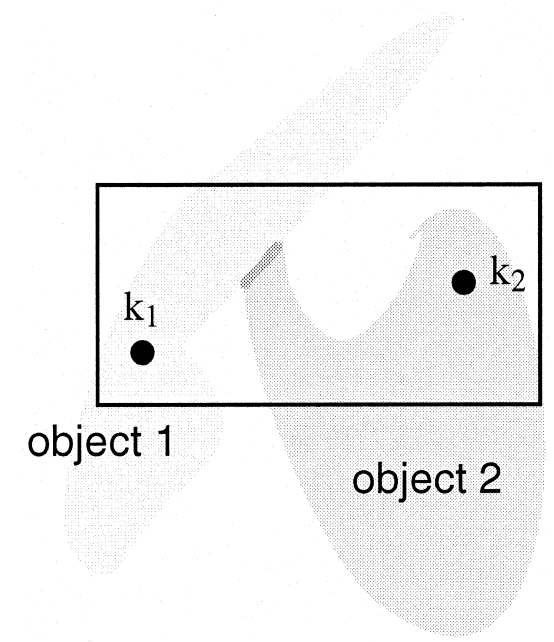

Fig. 10. Separating objects when the location of the boundary is unknown. 


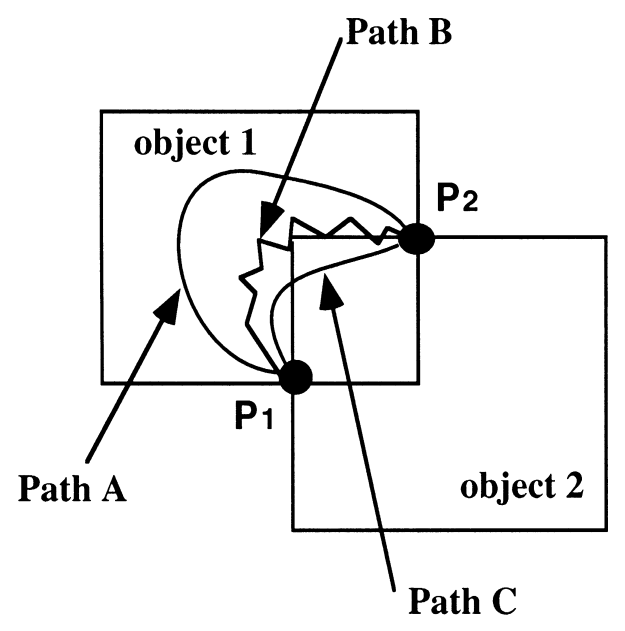

Fig. 11. Path restriction.

that we have considered so far, this function is simply the identity

$$
y_{i}=F\left(x_{i}, \mathrm{X}\right)=x_{i} .
$$

However, if the input image is a texture image, the feature extraction function will be more complex. In particular, it will always be nonlinear and take into account the neighboring pixels.

\subsection{Restricting path direction and length}

Between two points, there can be many paths. Consider the example in Fig. 11. Assume two end points are given and three paths could be found by the algorithm (paths A, B, C). None of these might be a good boundary. This problem will occur when the boundary has no width and the image is noisy. An example of such images is shown in Fig. 12, along with its gradient. This kind of image is difficult to segment for any algorithm. However, if more information about the boundary is available, the connectivity-based threshold algorithm can use such information to restrict the path between the two points. A criterion that can be applied to any

(a)

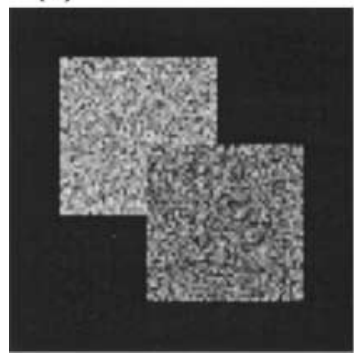

(b)

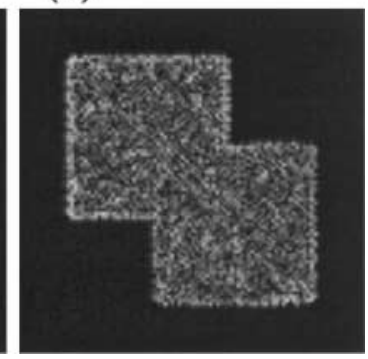

Fig. 12. Image with strong noise (a) and its gradient image (b). 


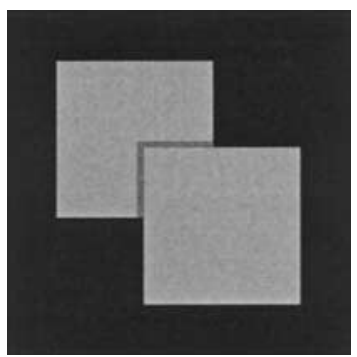

Fig. 13. Original uncorrupted image.

image is to take into account the length of the path. If the length is considerably longer than the distance between the two points, it is most likely that the path is not the true boundary. By this restriction, path A in Fig. 11 can be eliminated. Another restriction is the smoothness of the path. If the path has many sharp turns like path B in Fig. 11, it is unlikely to be the true boundary unless there is a strong reason to believe that the boundary has many sharp turns. By this restriction, path $\mathrm{B}$ in Fig. 11 can be eliminated. There can be other heuristic restrictions which can be used to determine whether or not the path is the true boundary. If such information is available, it can be effectively used to guide the connectivity-based threshold algorithm.

\section{Experiments}

\subsection{Experiments with generated data}

First, we tested the algorithm on simulated data. Our basic test image contains two squares of equal intensity which are separated by small boundary region, as shown in Fig. 13. This configuration is intended to simulate the kind of problem that is the most frequently encountered in medical images, as illustrated in Fig. 2. It is also assumed that the two end points of the boundary are known. It is very easy to separate the two objects in such a uncorrupted image. So we added increasing amounts of noise. Fig. 14 shows the corrupted images with SNRs 14.0, 4.44, 1.93 and $0.00 \mathrm{~dB}$, respectively. Fig. 15 shows the corresponding

(a)

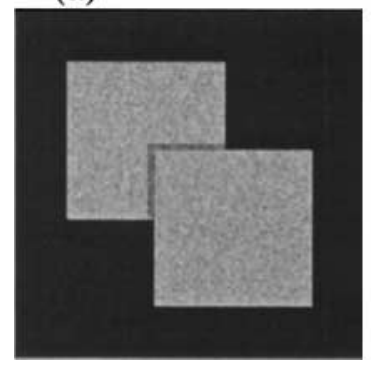

(b)

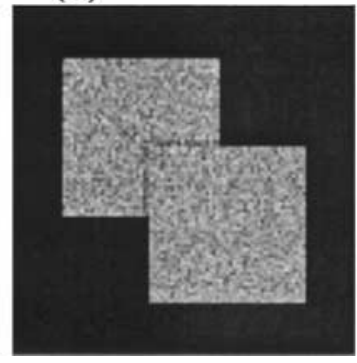

(c)

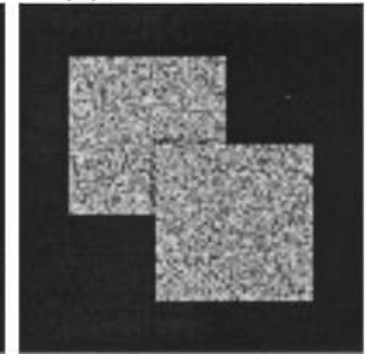

(d)

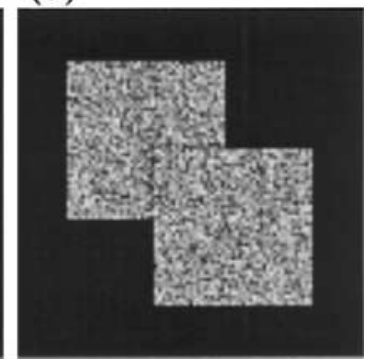

Fig. 14. Corrupted images with SNRs (a) $14.0 \mathrm{~dB}$, (b) $4.44 \mathrm{~dB}$, (c) $1.93 \mathrm{~dB}$ and (d) $0.00 \mathrm{~dB}$. 
(a)

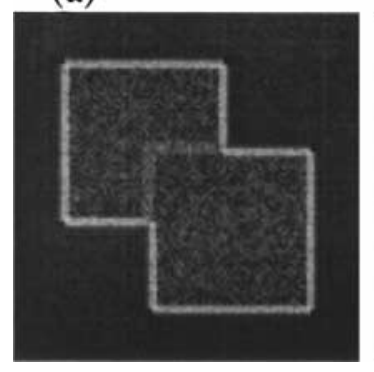

(b)

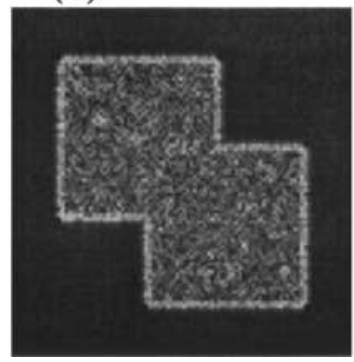

(c)

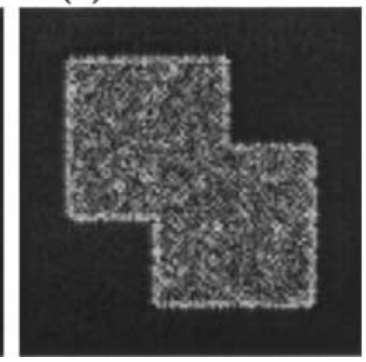

(d)

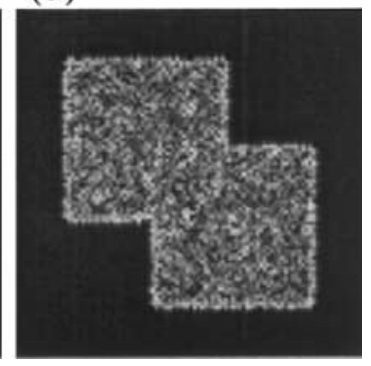

Fig. 15. Gradient images of the images in Fig. 14.

outputs of a gradient-based edge detector which obviously fails at detecting the boundary for $\mathrm{SNR} \leq 4.44 \mathrm{~dB}$. We applied the proposed algorithm to these images and Fig. 16 shows the results. When SNR is $14.0 \mathrm{~dB}$, the algorithm found the boundary almost perfectly. As the noise level increases, the boundaries extracted by the algorithm also become noisier. For SNRs of 4.4 and $1.93 \mathrm{~dB}$, the boundaries are still relatively close to the true one. The basic procedure breaks down at $0 \mathrm{~dB}$, which is not unexpected considering the amount of noise.

It is observed that the proposed algorithm can find a good boundary between objects even when a strong noise is present. The connectivity-based threshold segmentation algorithm performed well while segmentation based on gradient failed. Standard global threshold algorithms ( $k$-means) also fail for this particular example because the boundary region is too small and the two objects usually get lumped together.

\subsection{Experiments with real brain $M R$ images}

We have applied the connectivity-based threshold segmentation algorithm to brain MR images (Fig. 17). As mentioned in the introduction, the problem is to separate the brain from the surrounding structures. This segmentation task is a very important first step in analyzing and processing brain MR images; it is typically performed in a semi-automatic fashion. In our system which is fully automated, the images are first presegmented by applying a global threshold and removing all small regions and the skull. This preprocessing yields a binary segmentation map that at least includes the brain as a subpart. In most cases, this initial

(a)

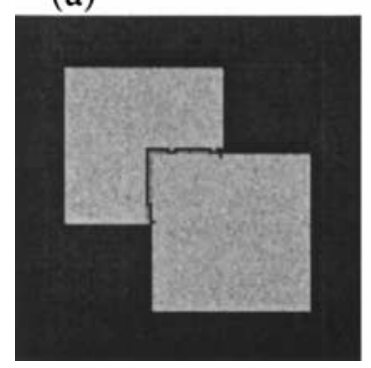

(b)

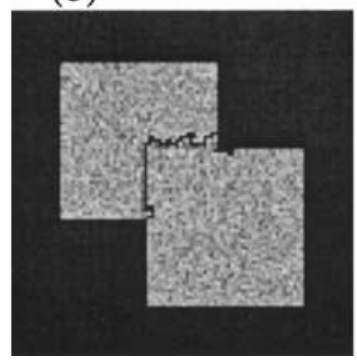

(c)

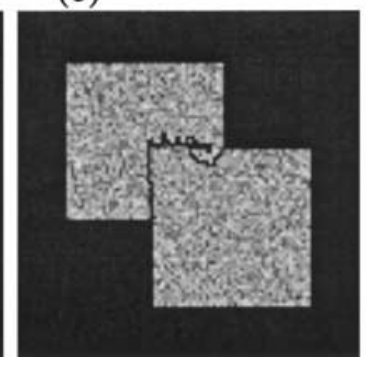

(d)

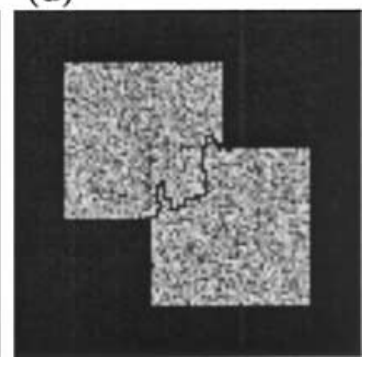

Fig. 16. Gradient images of the images in Fig. 14. 


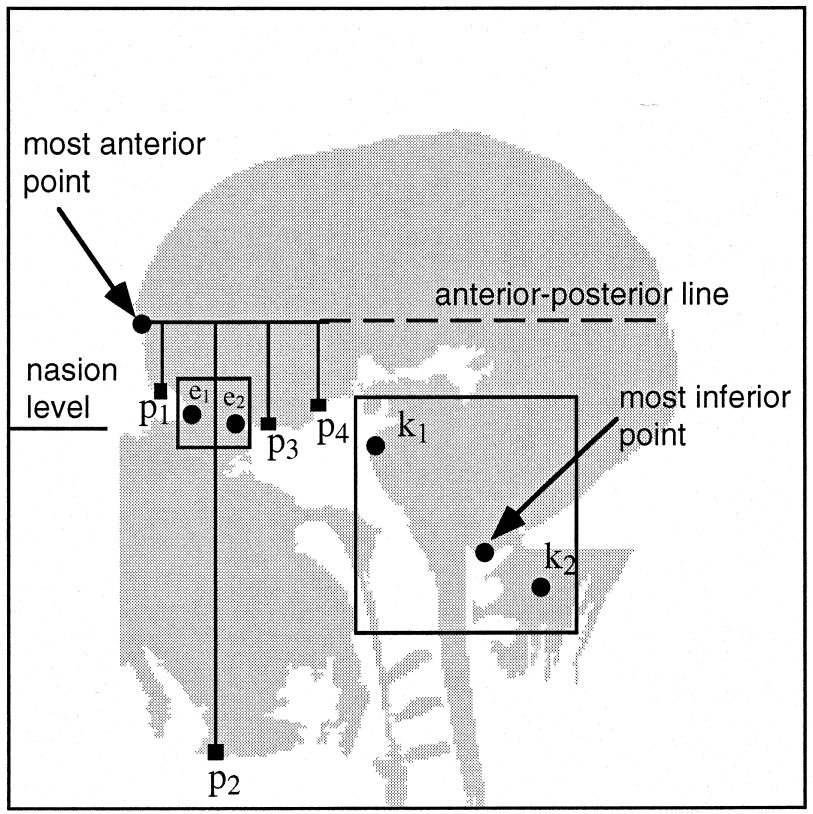

Fig. 17. Problem areas in segmenting brain MR image.

segmentation is imperfect because the brain may be connected to surrounding tissues as shown in Fig. 17.

Typically for sagittal images as shown in Fig. 17, there are two problematic areas. One is the inferior aspect of the forebrain mentioned previously, which is the most problematic region even when sagittal brain MR images are segmented manually or semi-automatically. To solve this problem, we first locate the most anterior point of the brain which is shown in Fig. 17. Starting at that level ( $\left.\mathrm{L}_{\text {anterior }}\right)$ we search downwards to find the boundary points at a uniform spacing, obtaining 4 points, $\mathrm{p}_{1}, \mathrm{p}_{2}, \mathrm{p}_{3}$ and $\mathrm{p}_{4}$ until we reach about half way along the anteriorposterior line. The points are displayed in Fig. 17 as black squares. Since we assumed that there is no brain below the nasion level, therefore if any of these points is below the nasion level, it indicates that the brain is connected to nonbrain tissues. If such a point is found, we find two valley points searching downwards from the $\mathrm{L}_{\text {anterior. }}$ The two valley points, $\mathrm{e}_{1}$ and $\mathrm{e}_{2}$, are shown in Fig. 17 as circles. In order to extract the boundary, we apply the connectivitybased threshold segmentation algorithm with the two end points known, $\mathrm{e}_{1}$ and $\mathrm{e}_{2}$ (procedure 1). In fact, we apply the algorithm to the pairs of points, $\mathrm{p}_{1}$ and $\mathrm{e}_{1}, \mathrm{e}_{1}$ and $\mathrm{e}_{2}, \mathrm{e}_{2}$ and $\mathrm{p}_{3}$, and $\mathrm{p}_{3}$ and $\mathrm{p}_{4}$ to ensure that there is no further connection between the brain and nonbrain tissues. Note that all these points are located automatically without any user intervention.

The other typical problematic area in Fig. 17 is in the posterior inferior brain and also tends to be connected to nonbrain tissues. Generally, it is more difficult to find reliable landmarks in this area of the brain. To solve this problem, we select two points, one $\mathrm{k}_{1}$ belonging to brain and the other $\mathrm{k}_{2}$ from a suspected nonbrain area. These two points are also selected automatically. The point $\mathrm{k}_{1}$ is relatively easy to select. The other point $\mathrm{k}_{2}$ is located as follows: first we find the most inferior point of the brain as shown in Fig. 17. If there is any region 


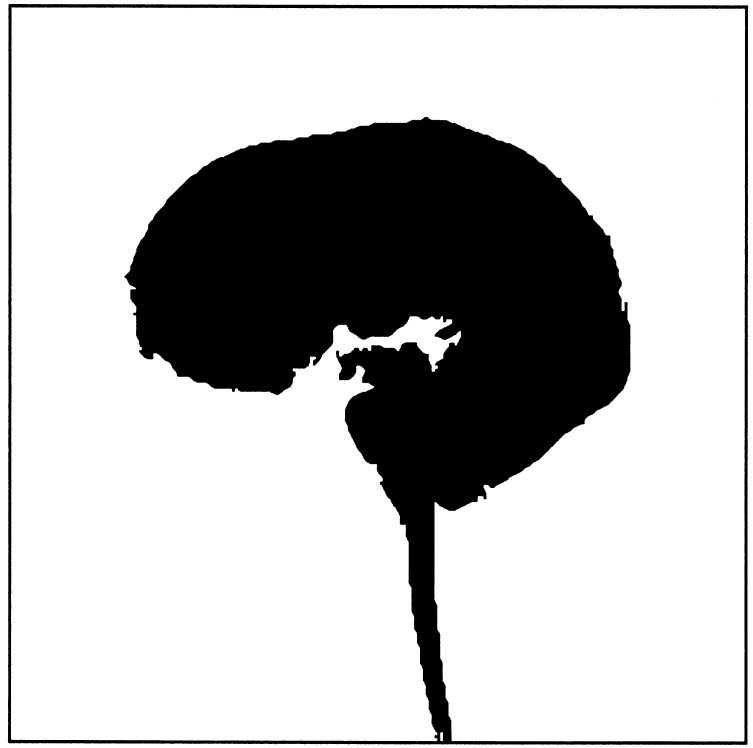

Fig. 18. Final result of the binary image.

below this point posterior to the brain stem, it is most likely that the region is not part of the brain and we select a point from it, i.e. $\mathrm{k}_{2}$. Then we apply the connectivity-based threshold segmentation algorithm with unknown boundary (procedure 2). Fig. 18 shows the final result of the binary image and Fig. 19 the corresponding the gray scale image. As can be seen, the algorithm generally finds a good contour of the brain.

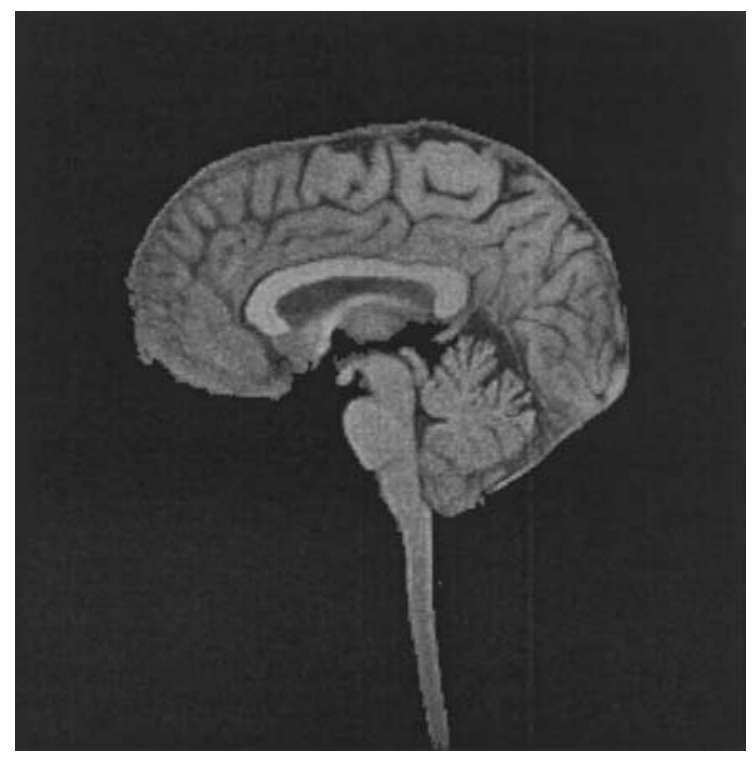

Fig. 19. Corresponding gray scale image. 


\subsection{Reliability of the proposed algorithm}

In order to demonstrate the reliability of the proposed algorithm, we have applied the algorithm to 120 midsagittal brain MR images. The original MR images and segmented images are shown in Appendix A. As expected, there are significant variations among the 120 images. For instance, there is much variation in the head area, from large (Fig. 20f, Fig. 23a) to small (Fig. 20i, Fig. 28g). Some of the subjects tilted forwards (Fig. 20j, Fig. 28e), some tilted backwards (Fig. 23j). In some images, artifacts from neighboring slices are included (Fig. 20f, Fig. 25d and f). As can be seen in Fig. 20g and Fig. 25e, sometimes there appear bright spots in the left-bottom corner of the forebrain, which caused error initially in some cases.

Although the proposed algorithm works relatively well, the algorithm initially produced some unsatisfactory results. After investigating the problems, we found that some of the initial assumptions were not always correct. For example, initially we assumed that the nose would be the leftmost point in the midsagittal brain MR image. This assumption was not valid in Fig. 26e, resulting in an error in locating the nasion. This problem was solved by putting additional restriction in the location of the nasion (the vertical difference between the nasion and the top of head should be smaller than 150 pixels). The bright spot in the left-bottom corner of the forebrain is taken care of by applying a threshold. There were other minor technical problems, all of which we were able to solve.

Although the proposed algorithm produces very satisfactory results in most cases, some of the segmented images still include nonbrained tissues. For instance, small nonbrain tissues are included under the inferior aspect of the forebrain (Fig. 21c, Fig. 22c, Fig. 28h). Some of the images include meninges (brain covering) which need to be excluded depending on applications. However, considering the size of test images and the large variations among the images, it seems that the proposed algorithm is quite reliable and will be very useful for many applications.

\section{Conclusion}

Stripping away of nonbrain tissues is crucial in processing brain MR images and the first step for various analyses. In this paper, we proposed a new segmentation algorithm which can be used for the development of a fully automated segmentation system. We have shown that this algorithm can automatically remove most nonbrain tissues from midsagittal slices. In order to develop a fully automated segmentation system, the algorithm must be applied to all slices of brain MR images. Presently, we have chosen to concentrate our attention on the middle slices which are typically the most difficult to segment due to the complexity of anatomy. Once these have been successful segmented, it should be possible to use this information to simplify the processing of the more lateral sagittal slices to yield a fully automated whole brain segmentation system.

The proposed connectivity based threshold segmentation algorithm may be useful for other applications. Although the method requires some prior information, the algorithm has some definite advantages. Firstly, it always finds a complete boundary between regions. Unlike edge detection methods, it does not have problems with broken or missing boundaries. Although the 
contour found by the proposed algorithm may not always be the true boundary, experiments showed that it is reasonably close to the true boundary in most cases. Secondly, the connectivity based threshold segmentation algorithm is shown to be robust even in the presence of strong noise. Thirdly, if some additional information about the boundary is available, it can be easily used to improve the performance. In particular, we have demonstrated that it can be used to segment the brain from surrounding tissues in MR images. The method can also be applied interactively to improve the results obtained by other segmentation algorithms. Regions that have been improperly merged can easily be separated if the user just specifies two points.

\section{Unlinked References}

[7-20]

\section{Appendix A}

For additional results using the proposed algorithm see Figs. 20-31. 
(a)

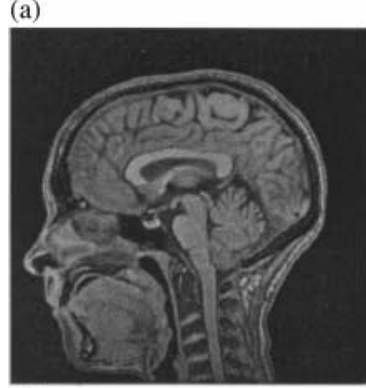

(c)

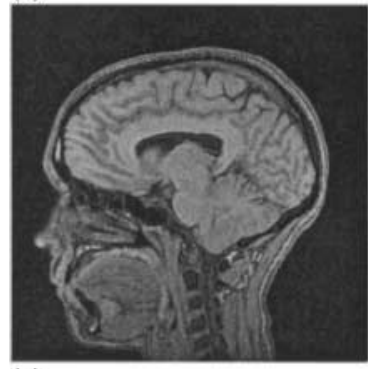

(e)

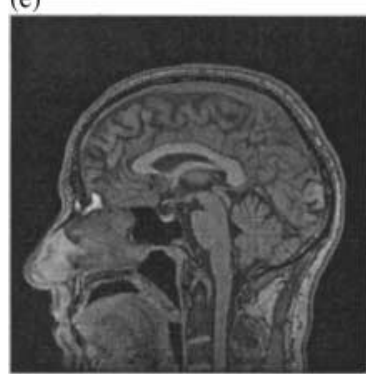

(g)

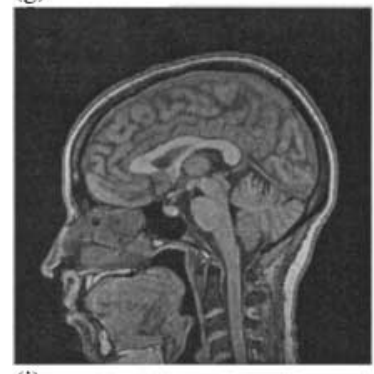

(i)

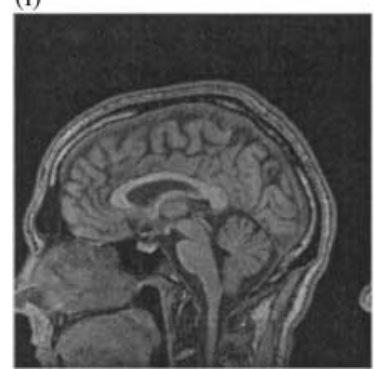

(b)
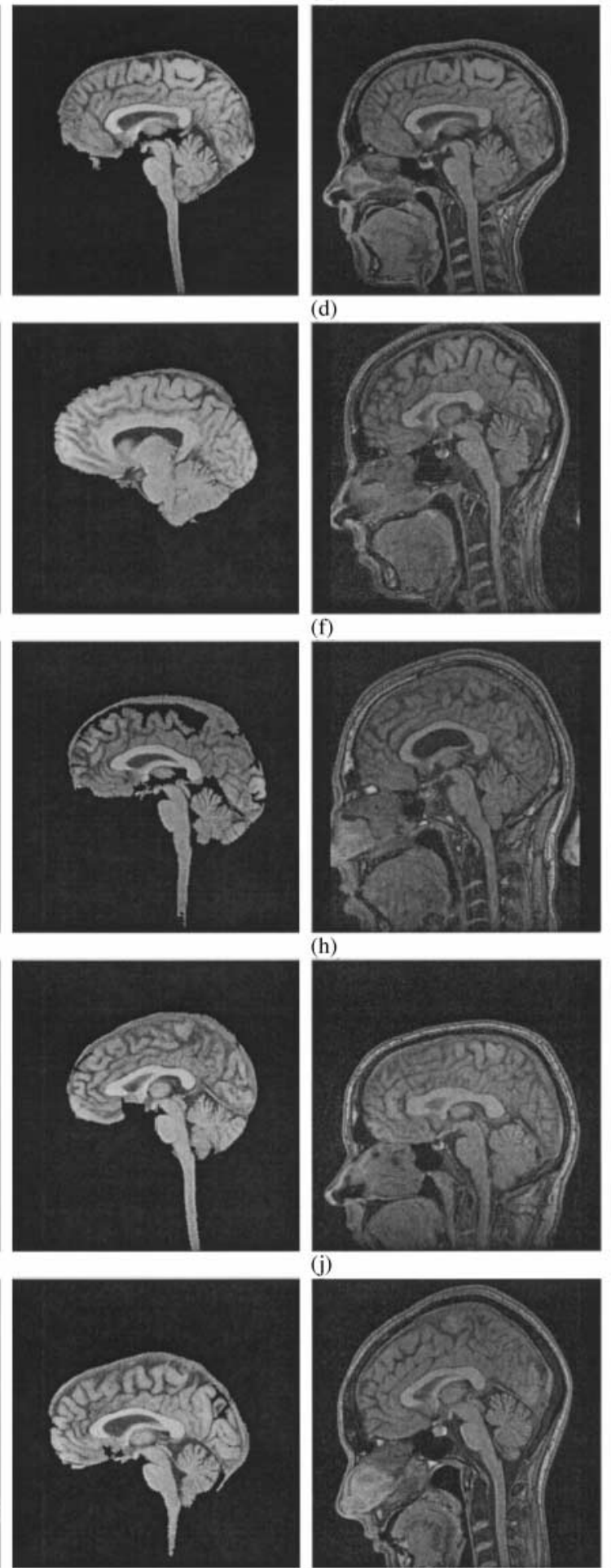

(h)
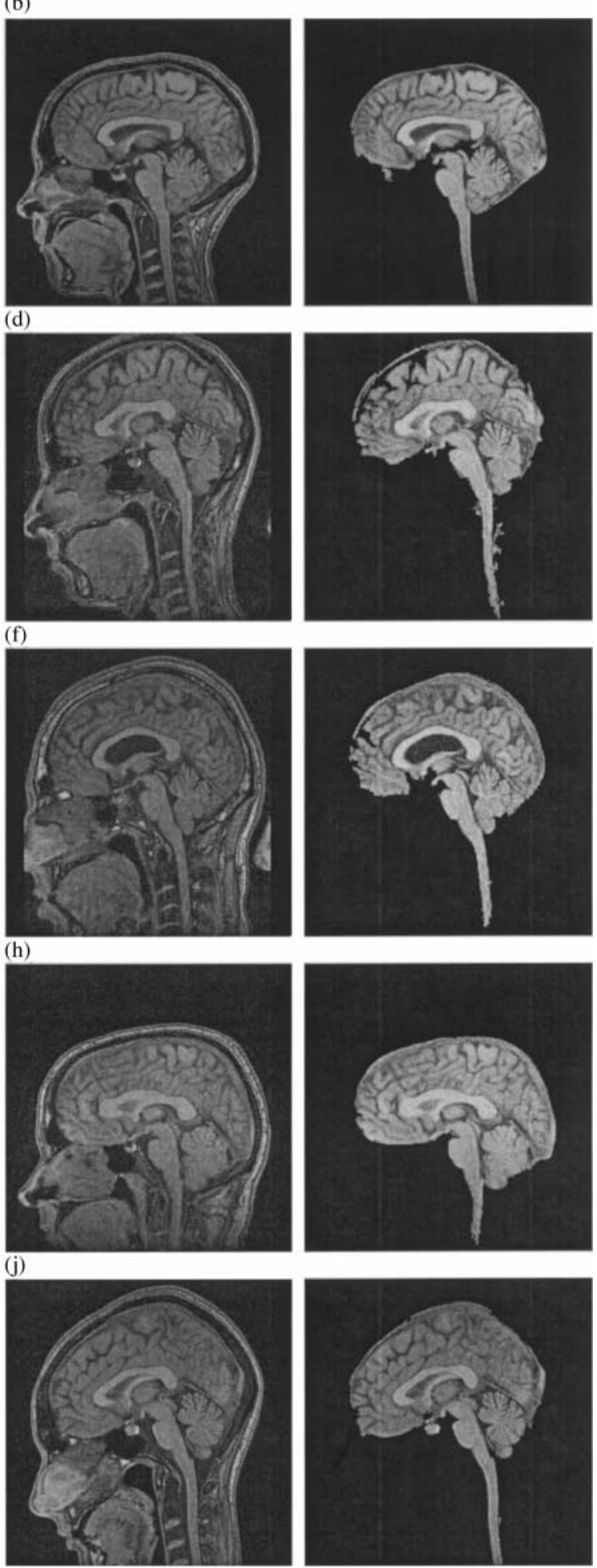

Fig. 20. 
(a)

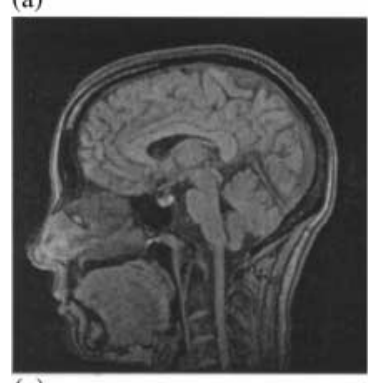

(c)

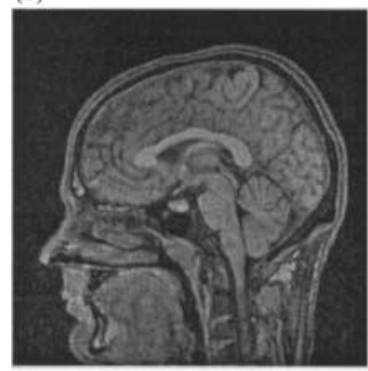

(e)

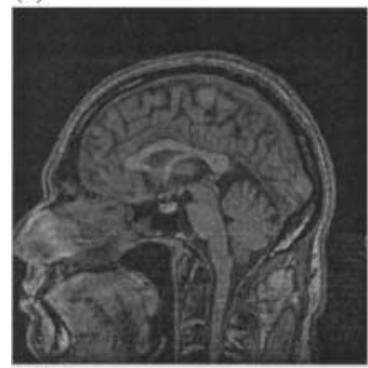

(g)

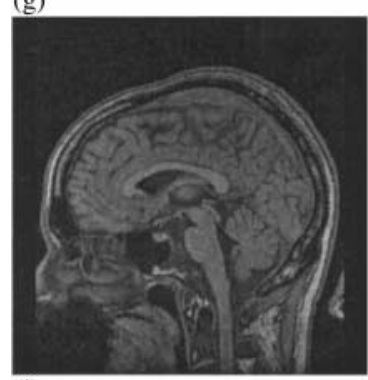

(i)

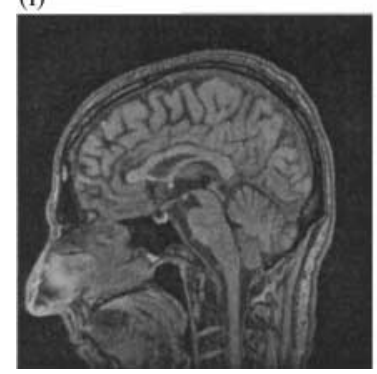

(b)
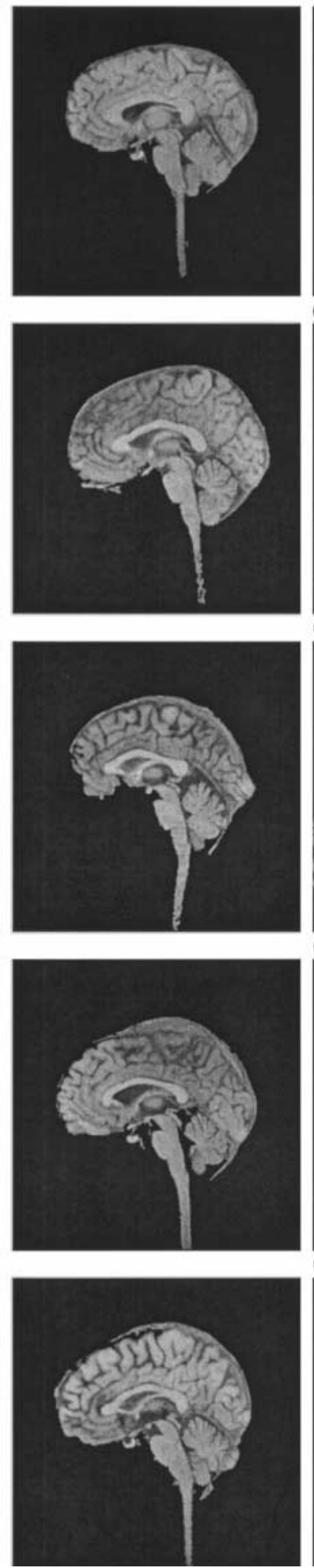
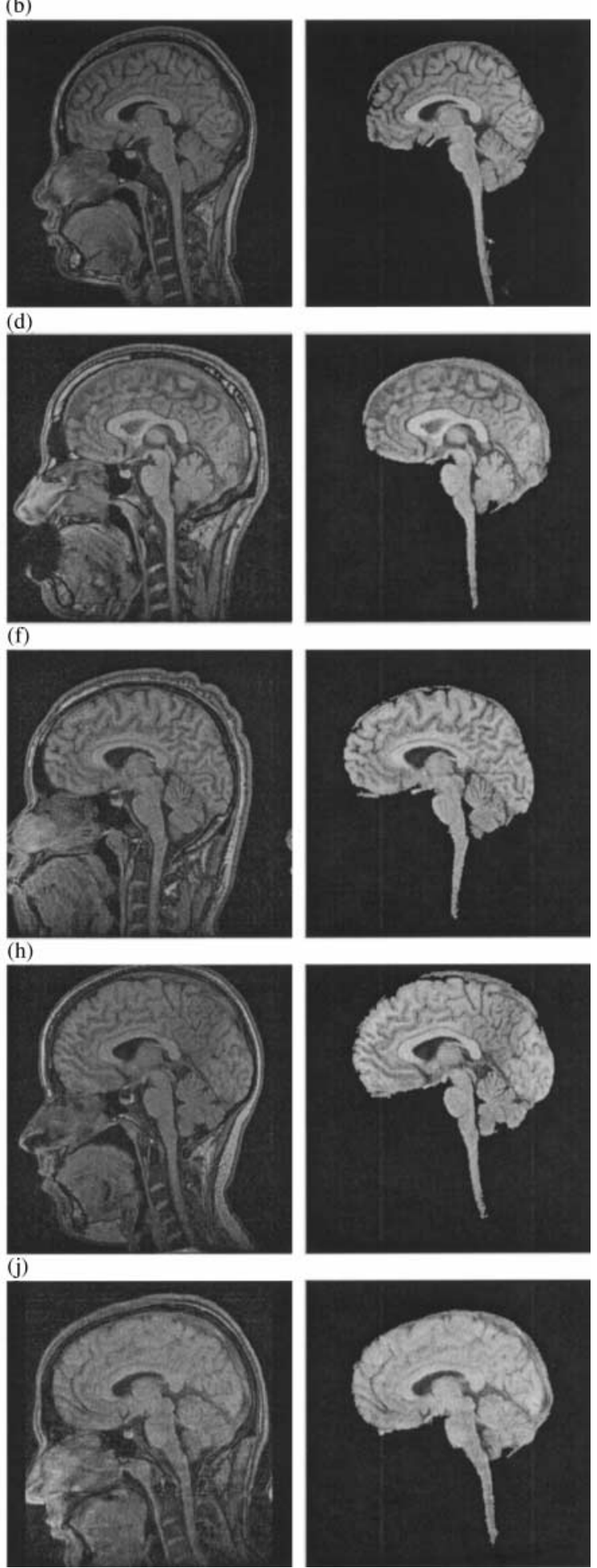

Fig. 21. 
(a)

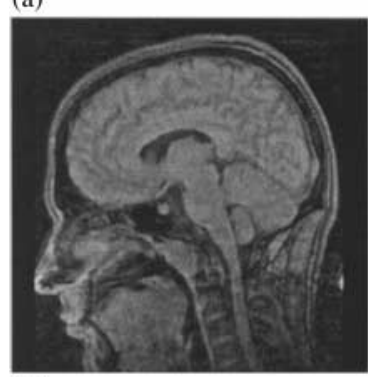

(c)

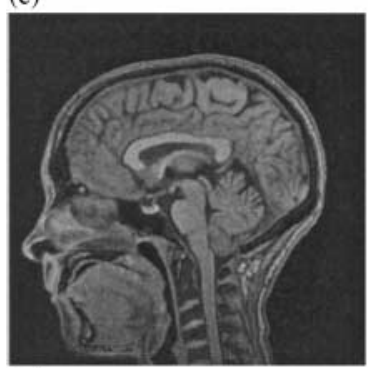

(e)

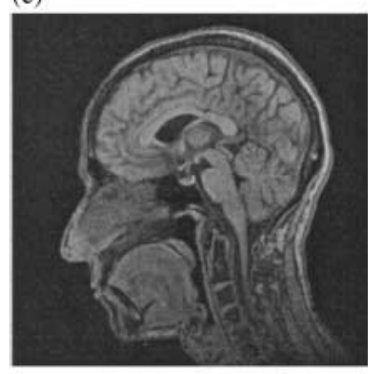

(g)

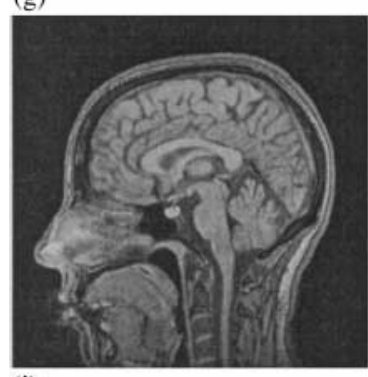

(i)

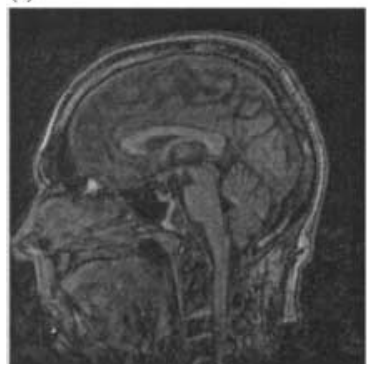

(b)
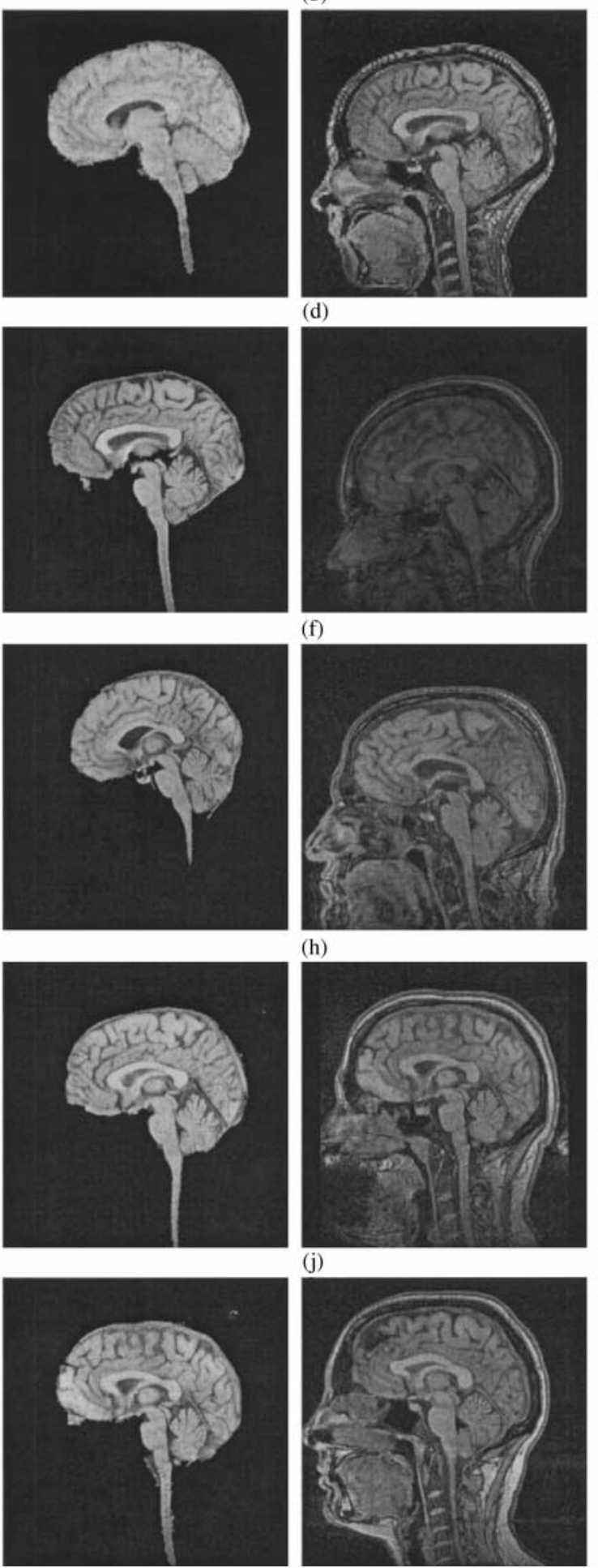

(d)

(f)

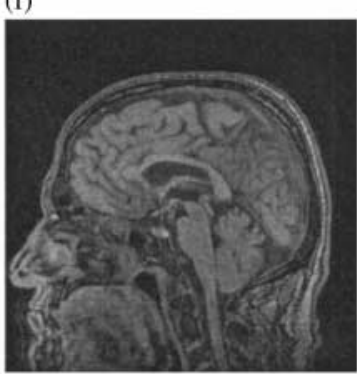

(h)
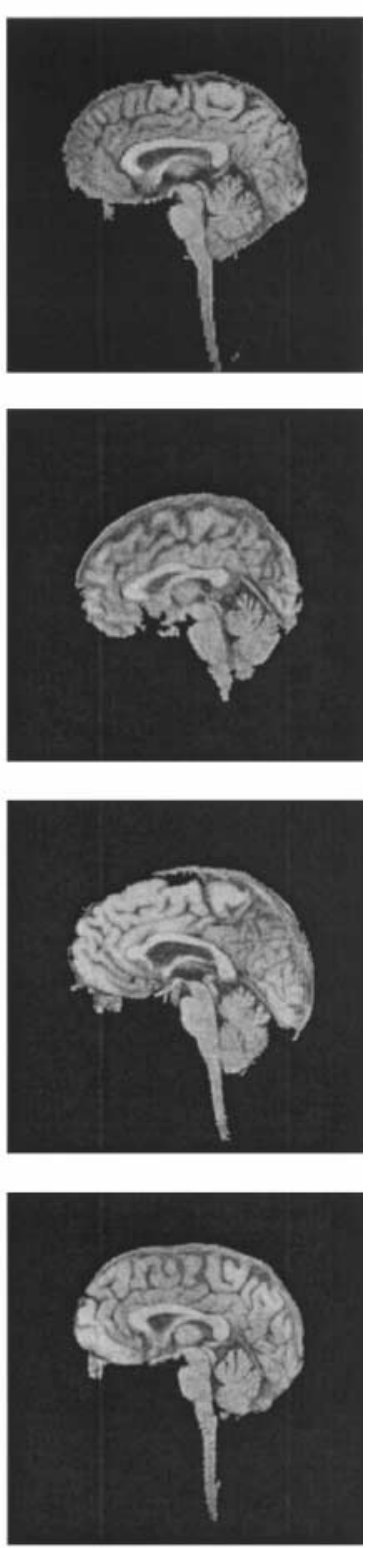

(j)
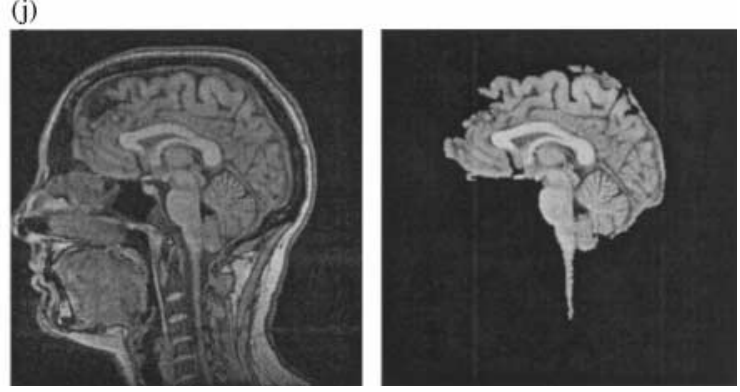

Fig. 22. 
(a)

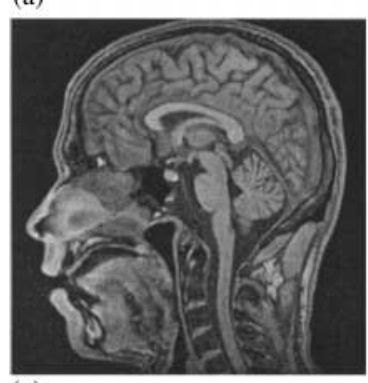

(c)

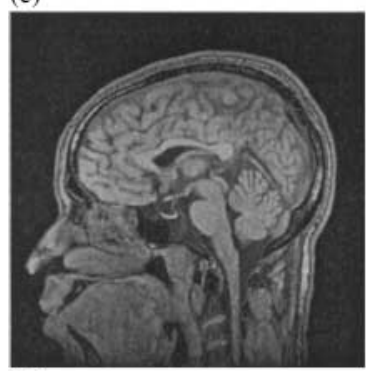

(e)

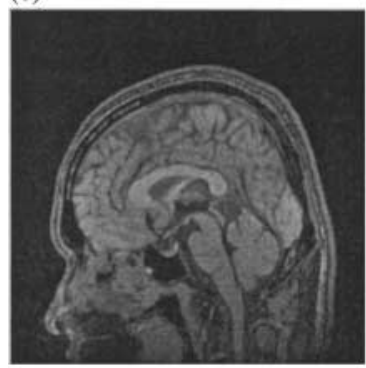

(g)

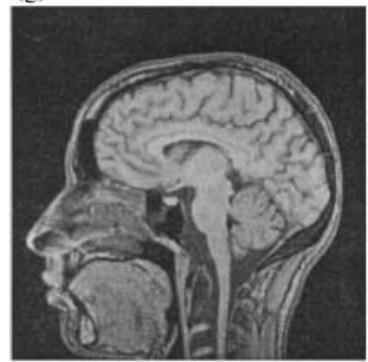

(i)

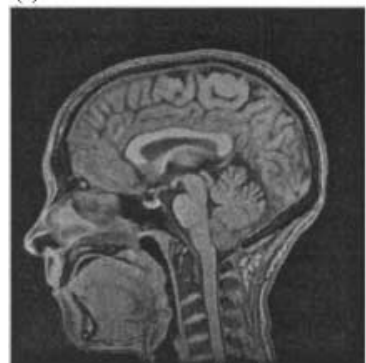

(b)
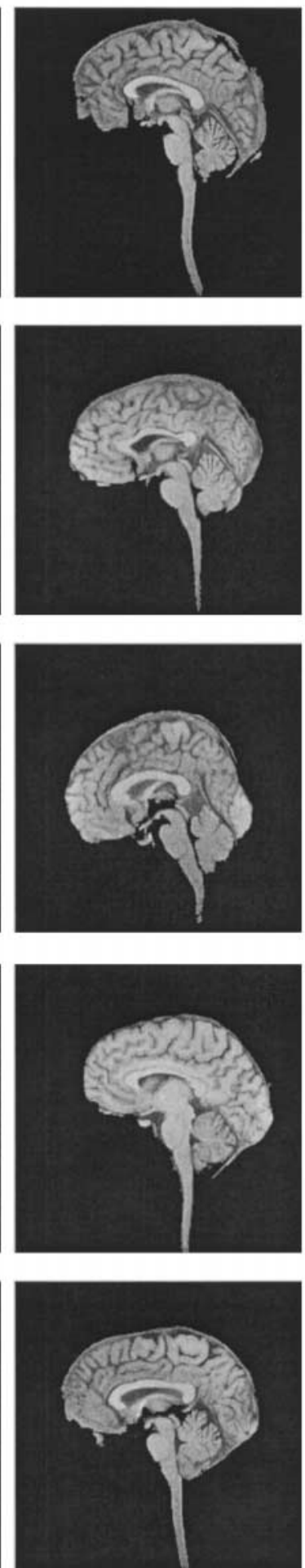

(d)

(f)

(h)
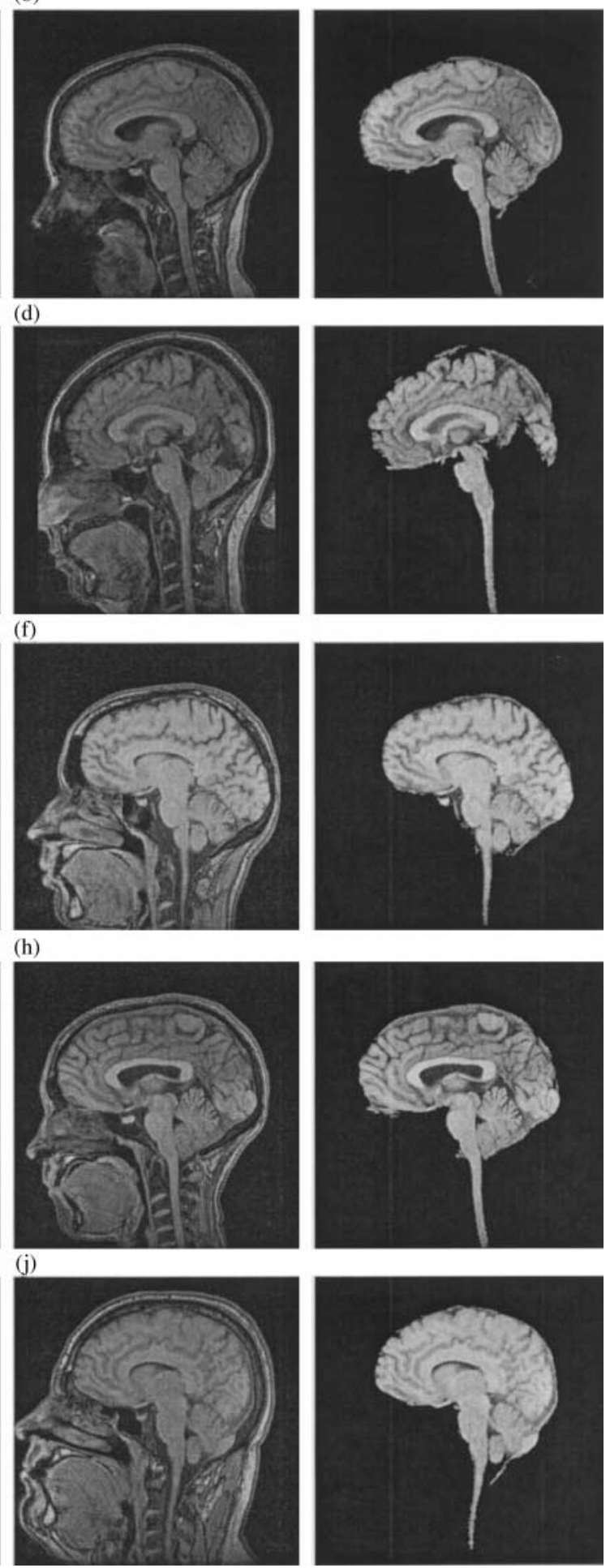

Fig. 23. 
(a)

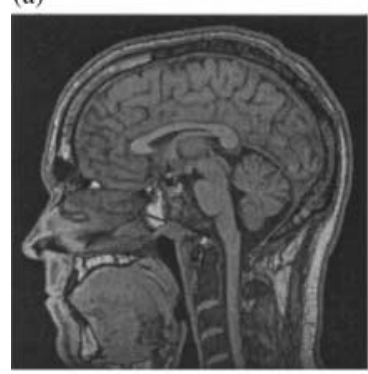

(c)

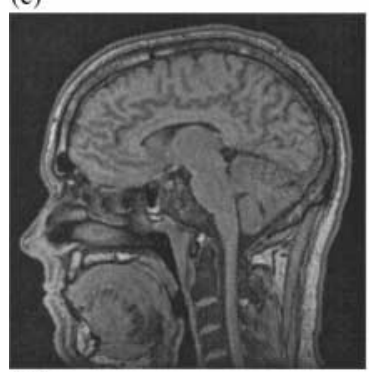

(e)

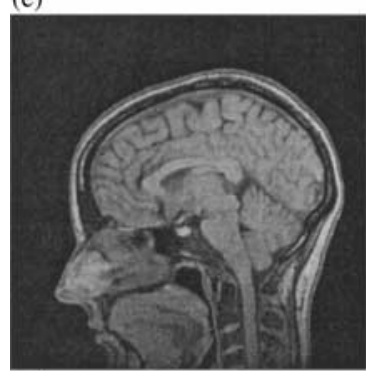

(g)

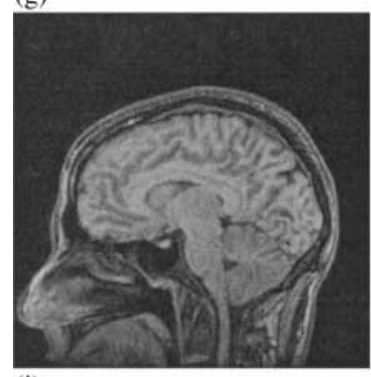

(i)

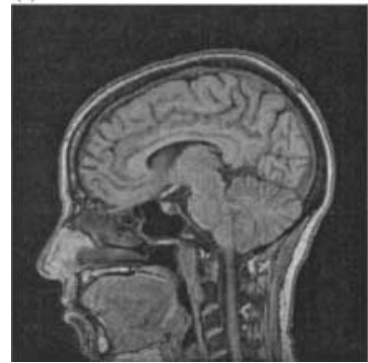

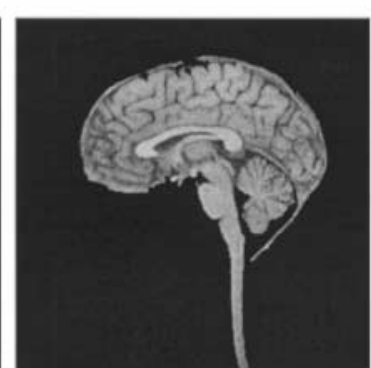

(b)

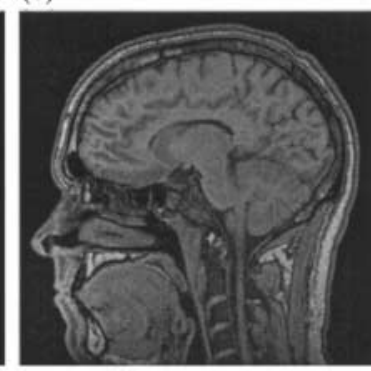

(d)
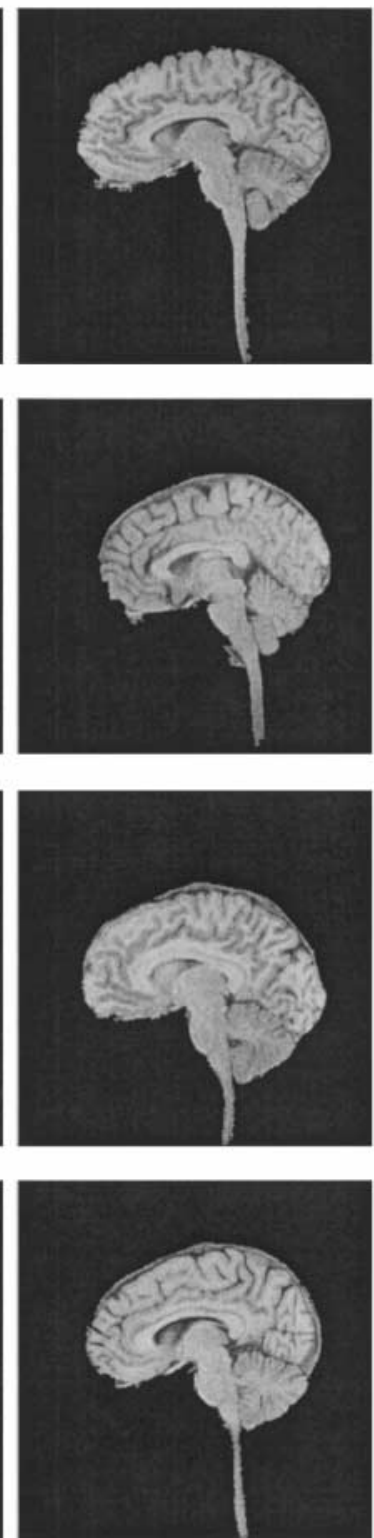
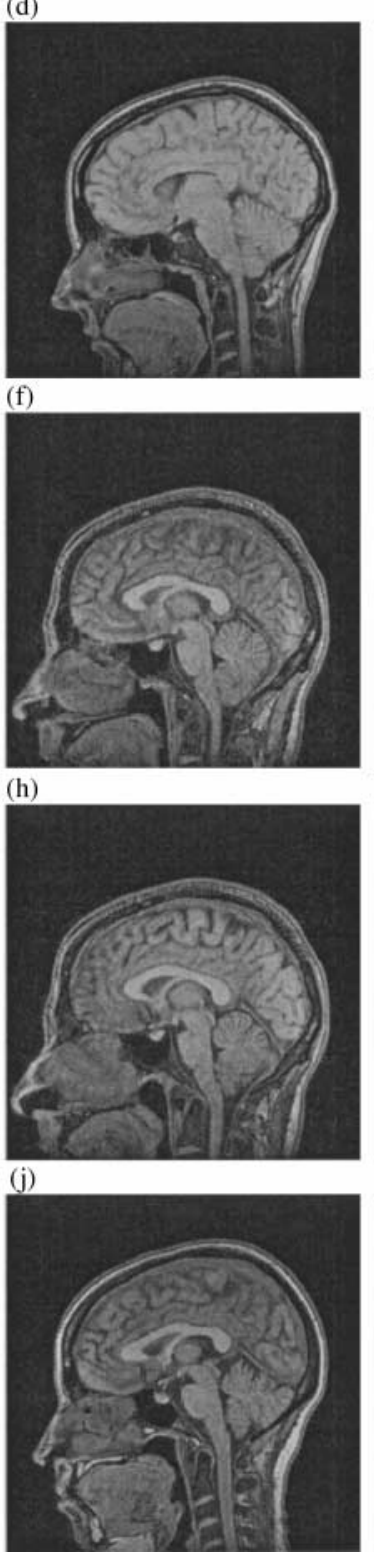
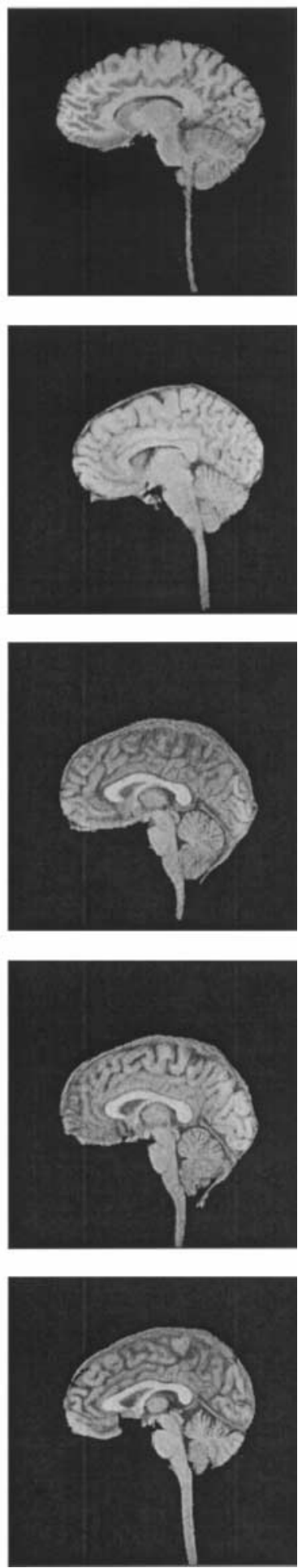

Fig. 24. 
(a)

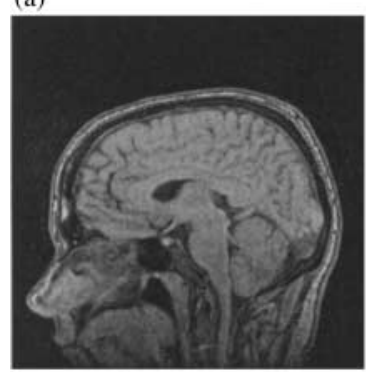

(c)

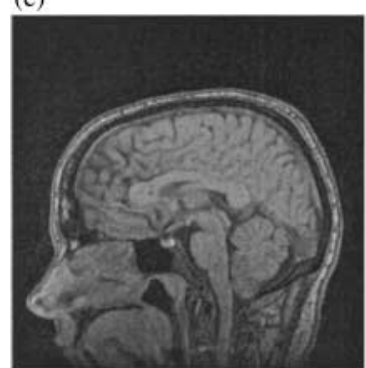

(e)

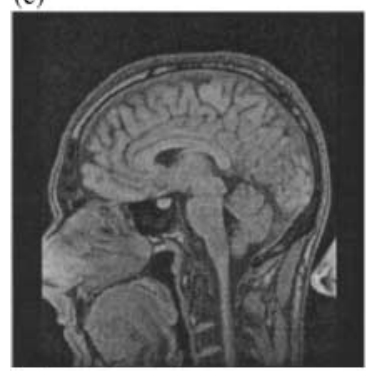

(g)

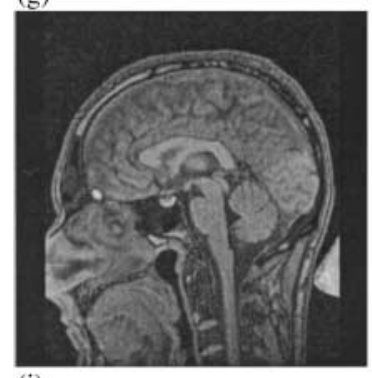

(i)

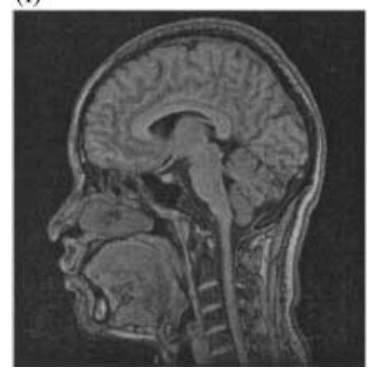

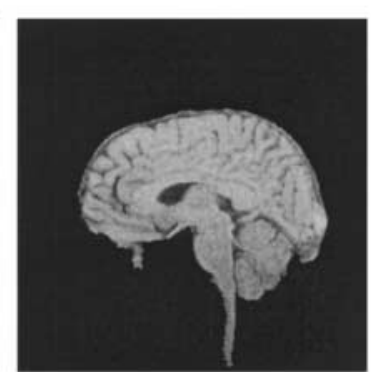

(b)

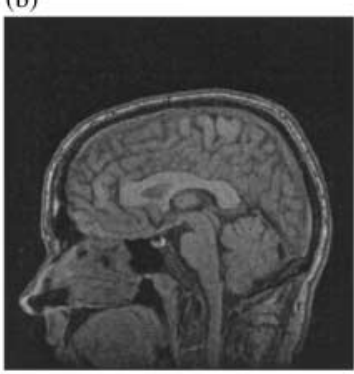

(d)
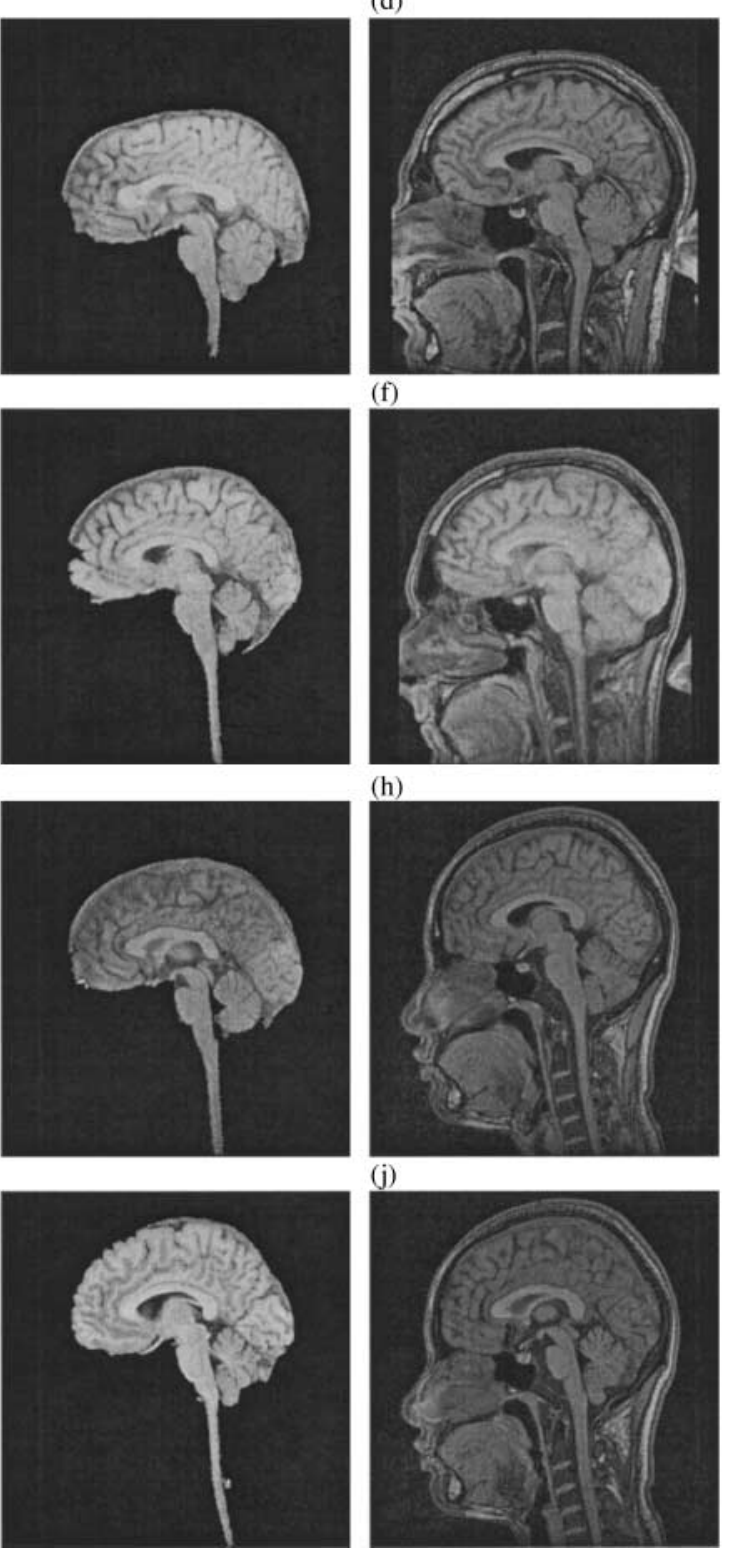

Fig. 25 .

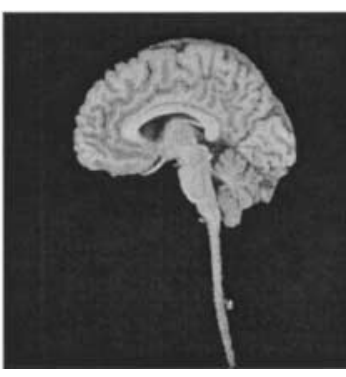

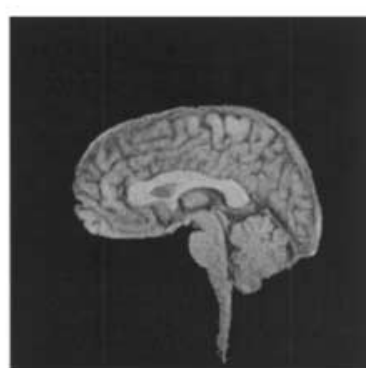
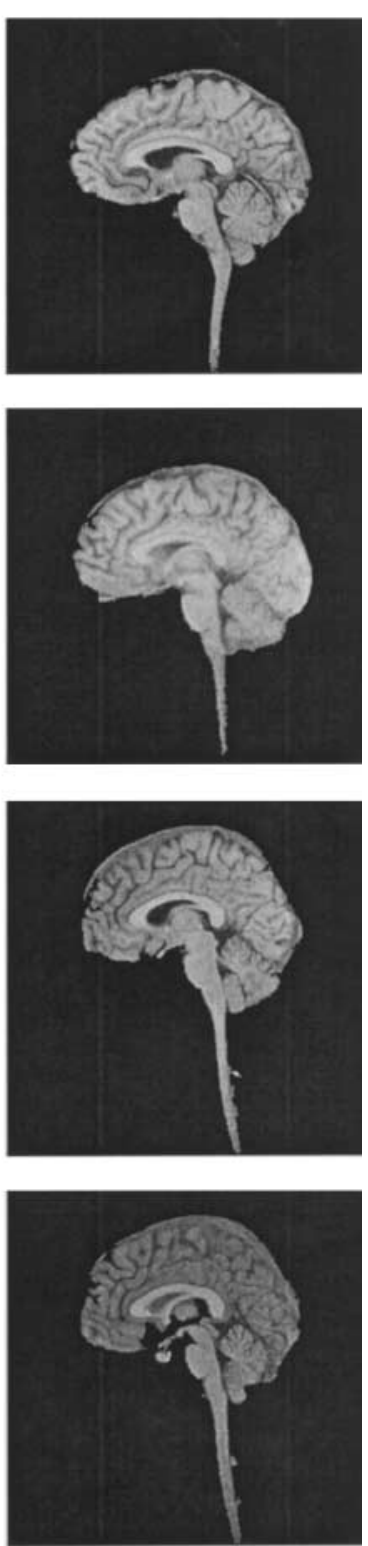
(a)

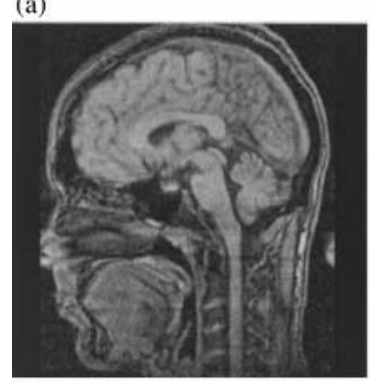

(c)

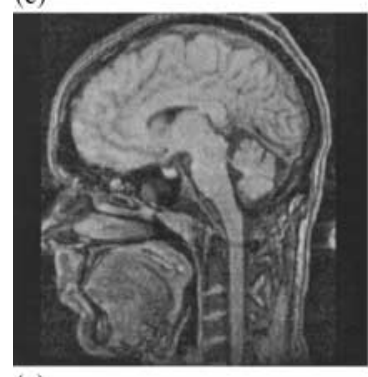

(e)

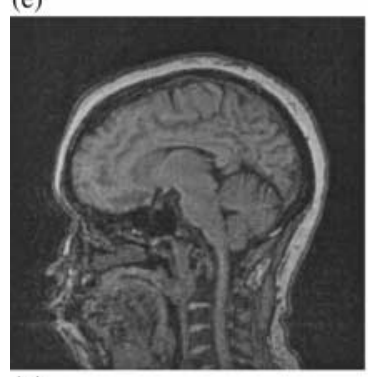

(g)

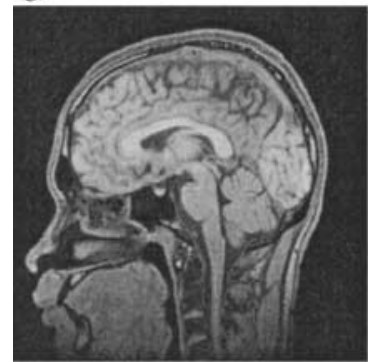

(i)

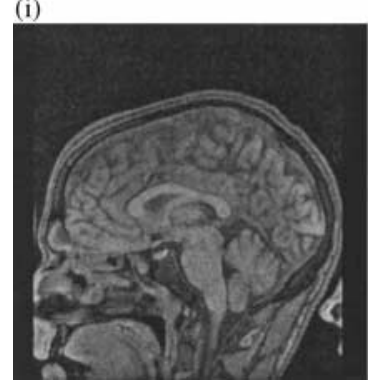

(b)
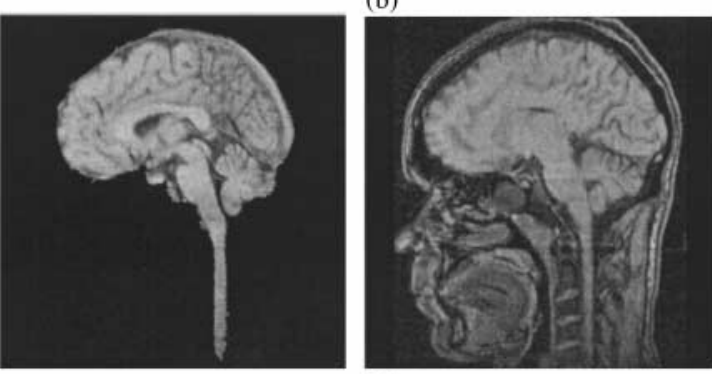

(d)
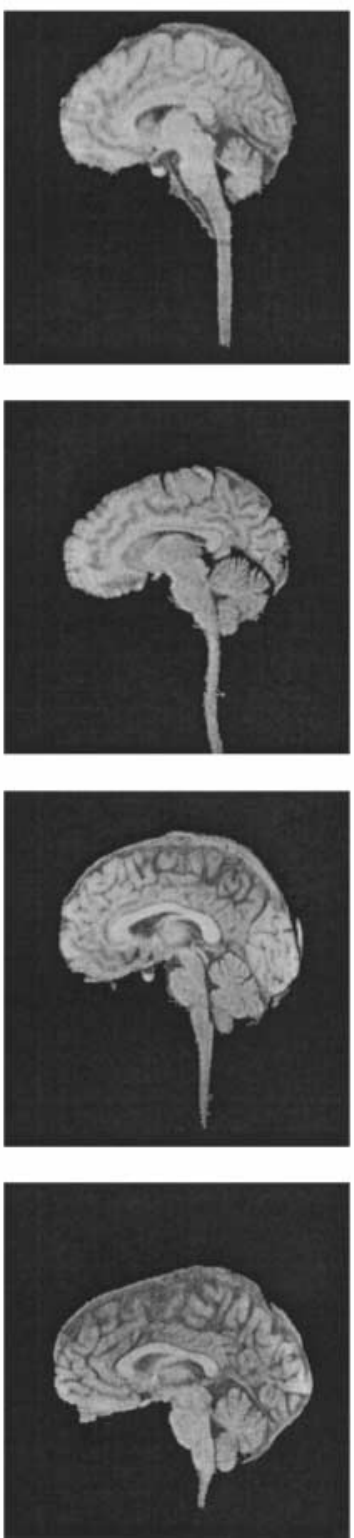
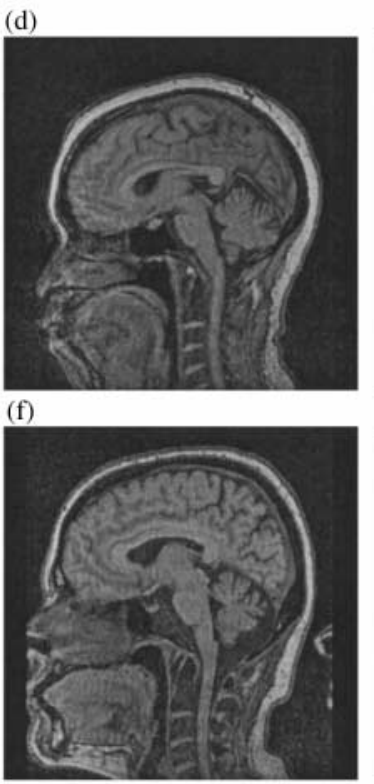

(h)
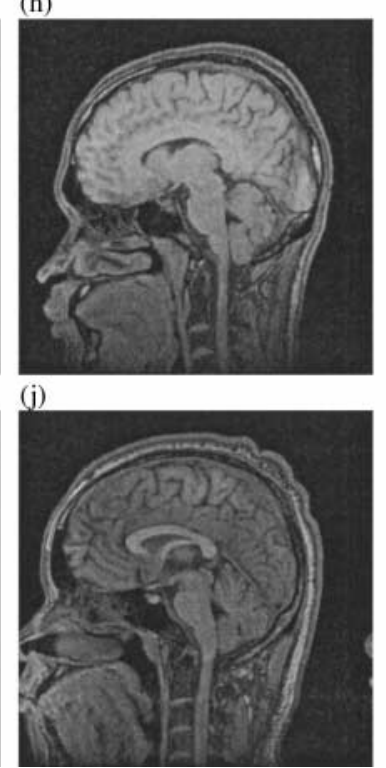
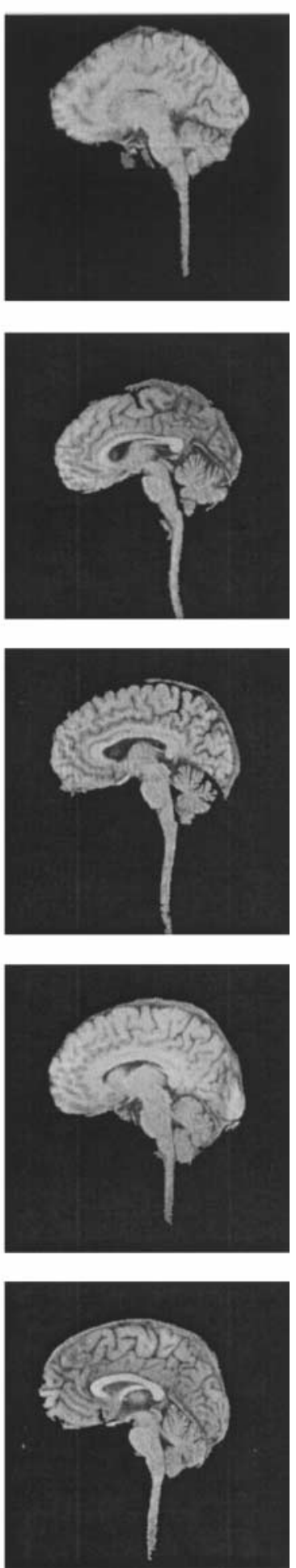

Fig. 26. 
(a)

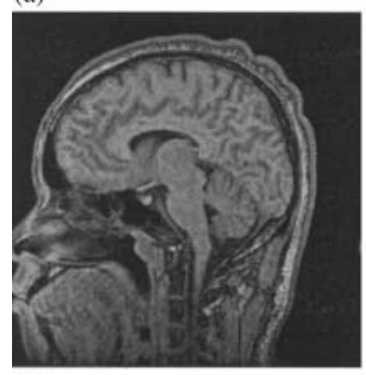

(c)

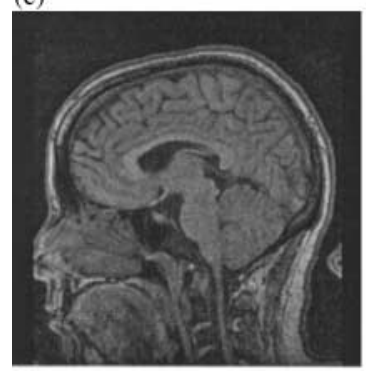

(e)

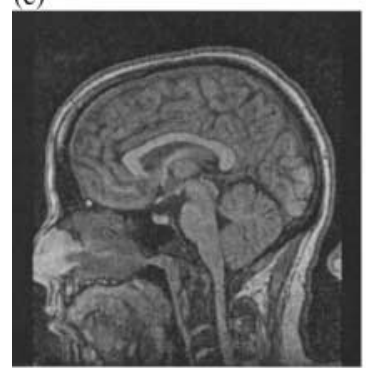

(g)

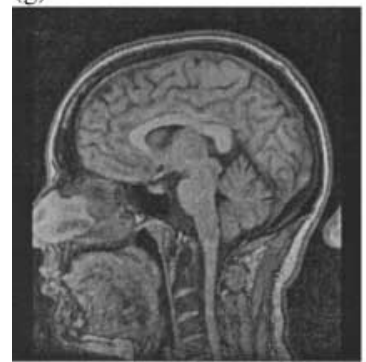

(i)

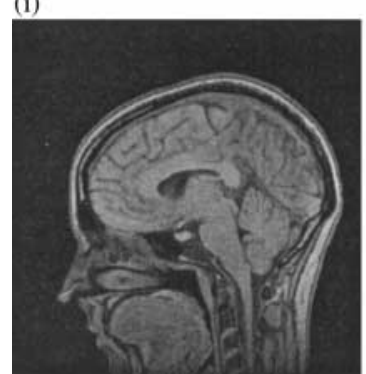

(b)
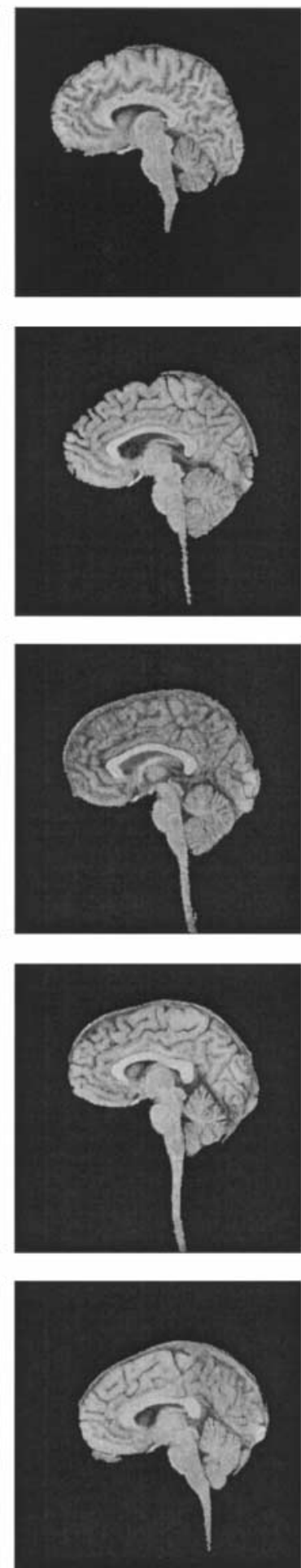

(h)
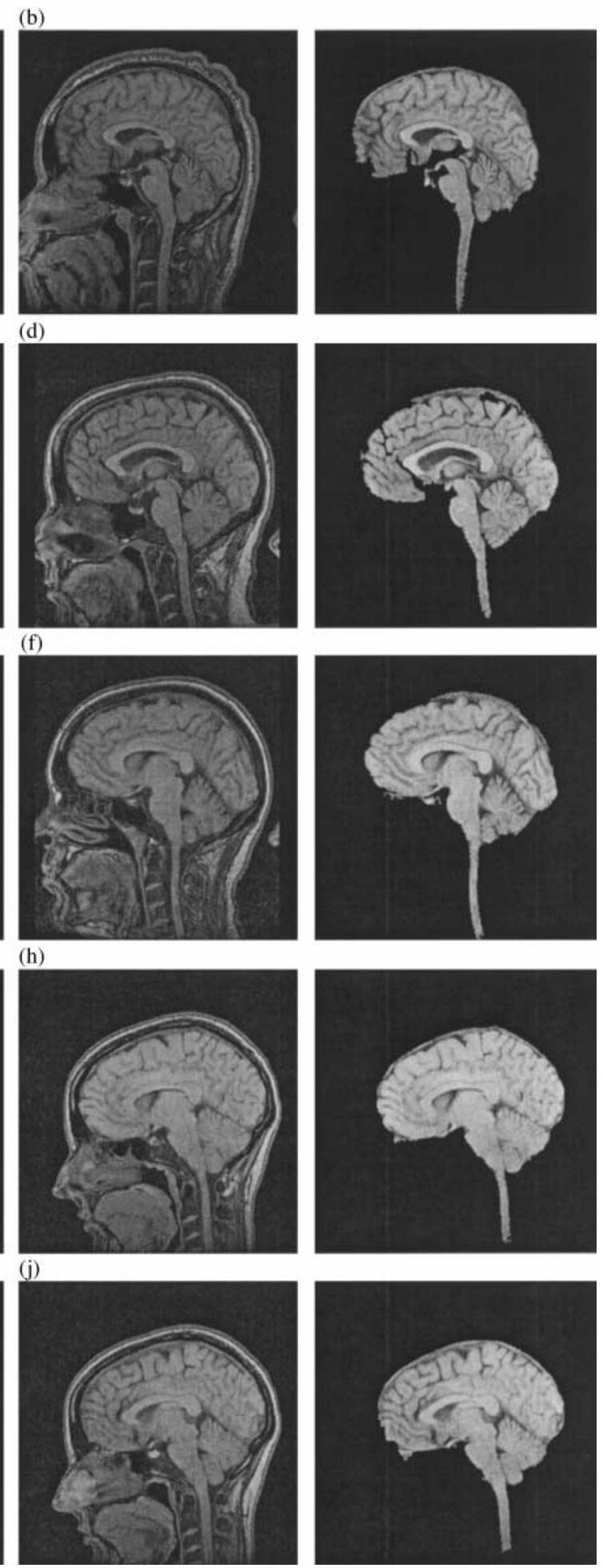

Fig. 27. 
(a)

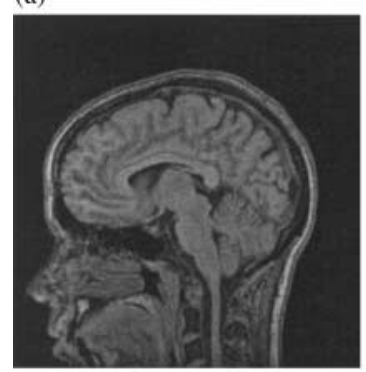

(c)

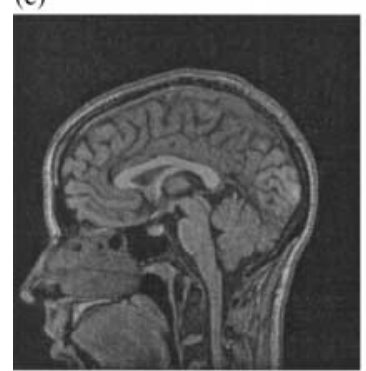

(e)

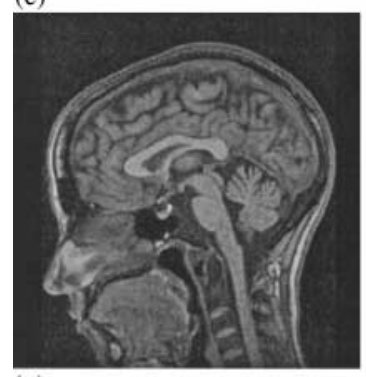

(g)

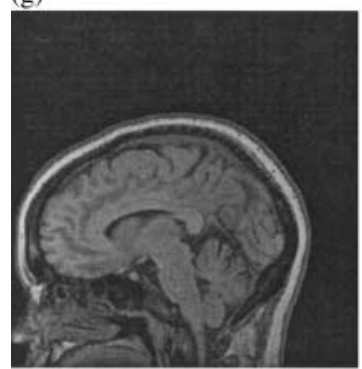

(i)

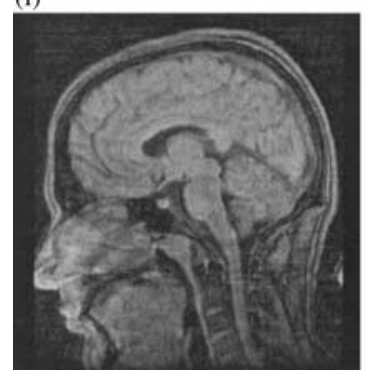

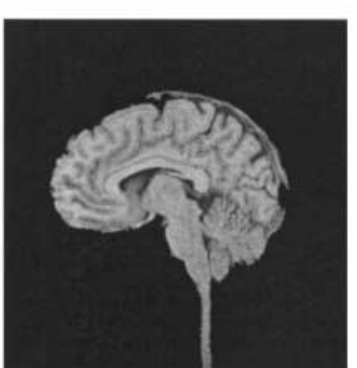
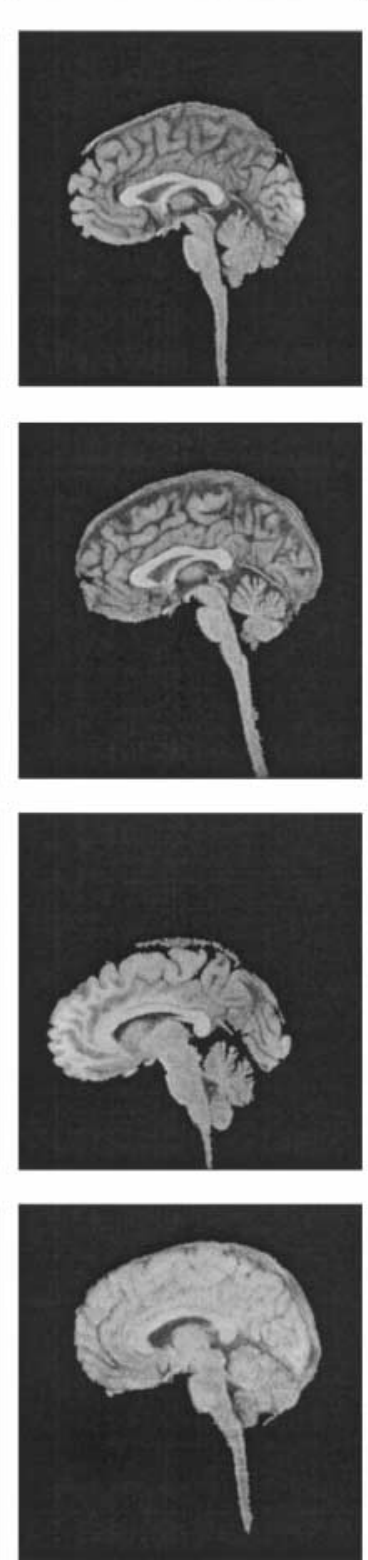

(b)

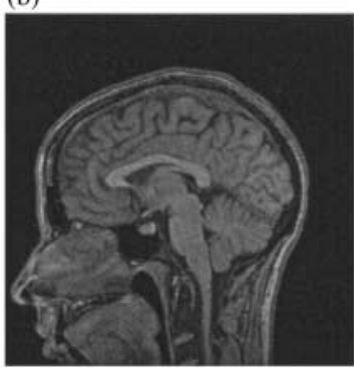

(d)
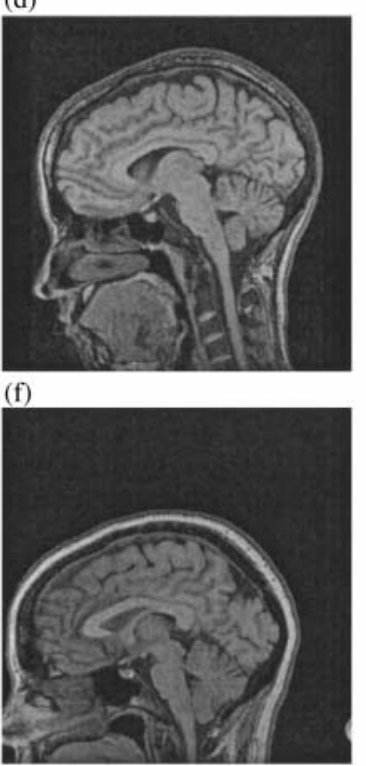

(h)
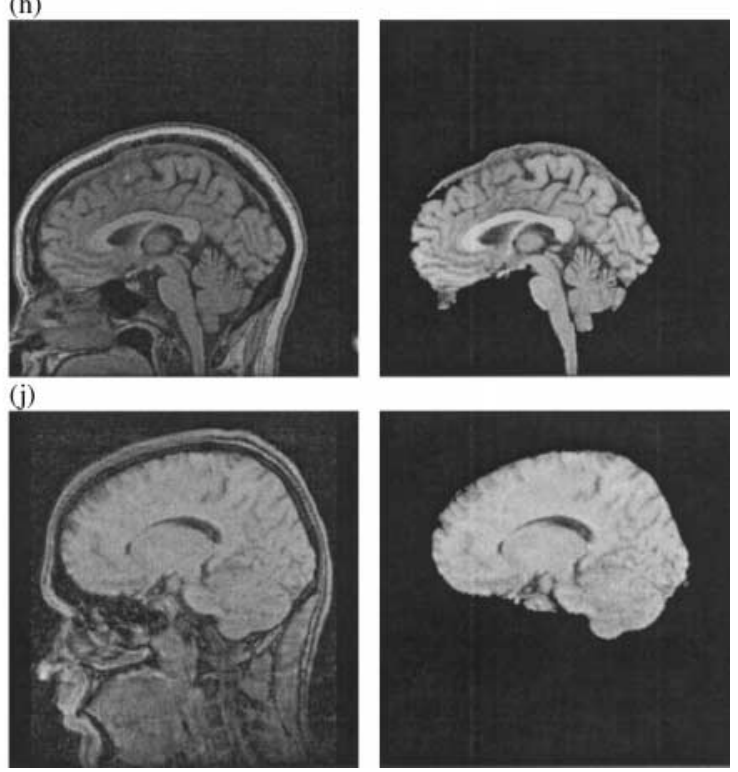

Fig. 28. 
(a)

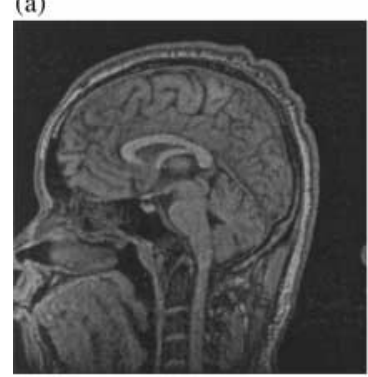

(c)

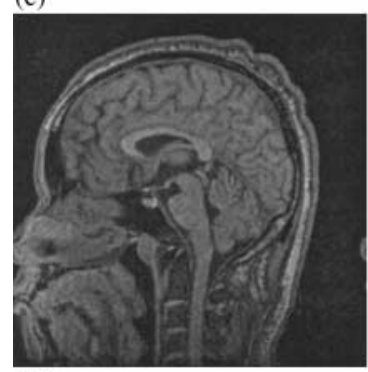

(e)

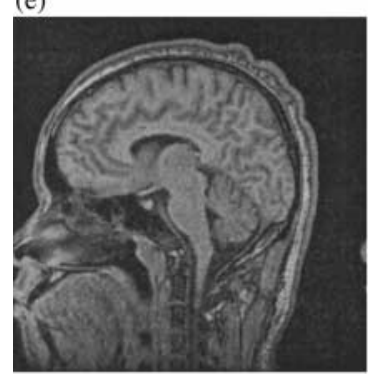

(g)

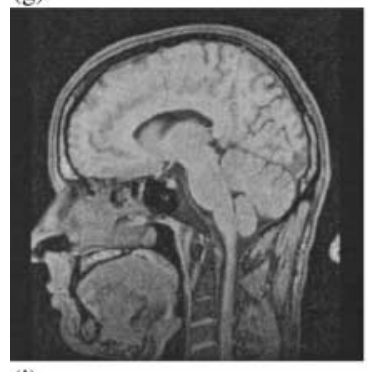

(i)

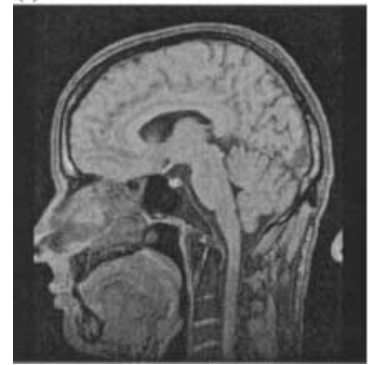

(b)
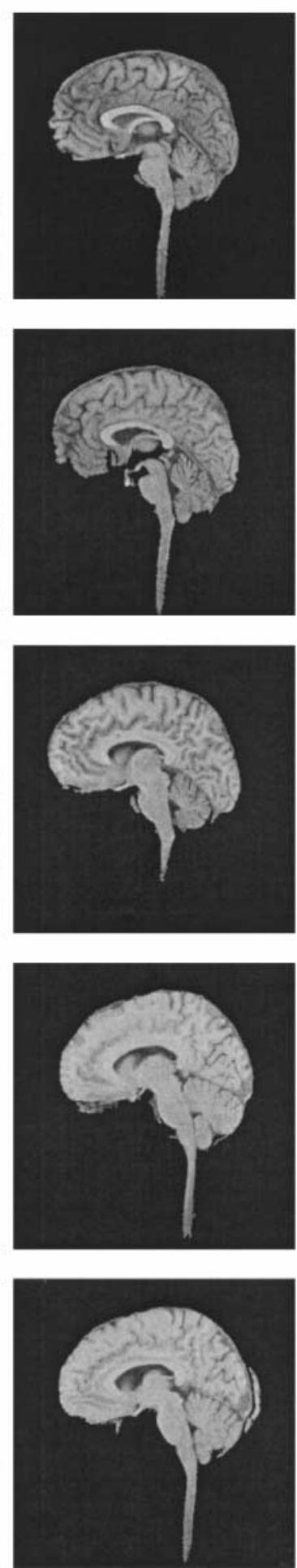

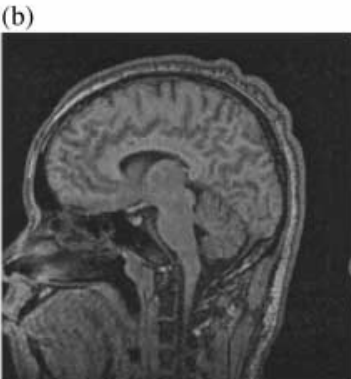

(d)
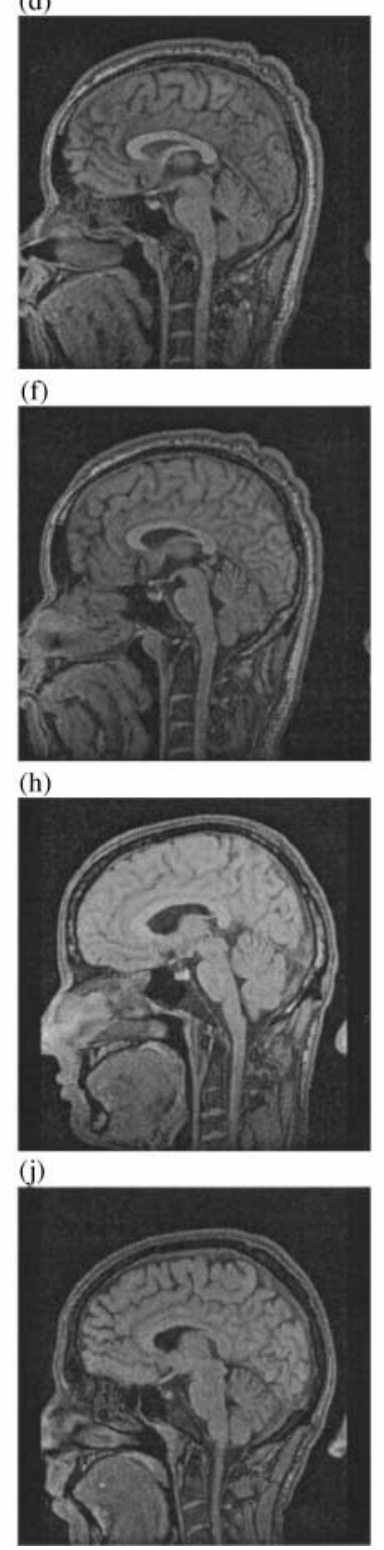
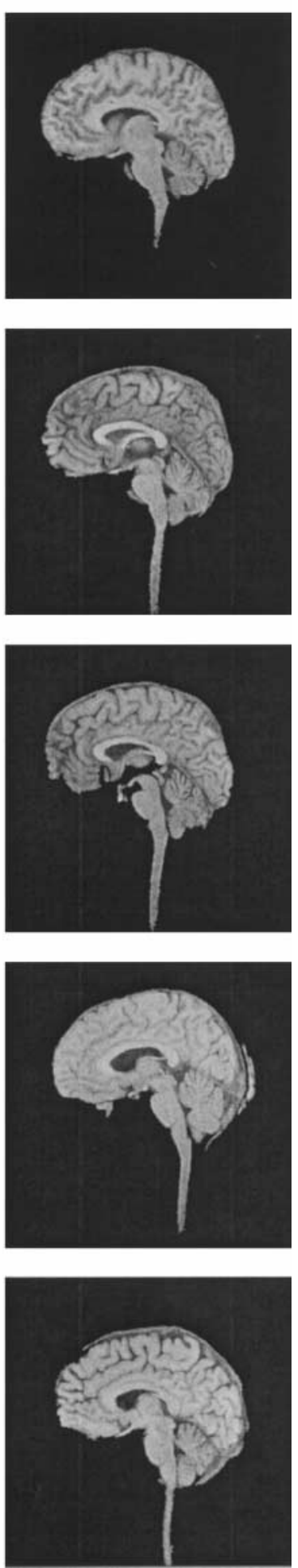

Fig. 29. 
(a)

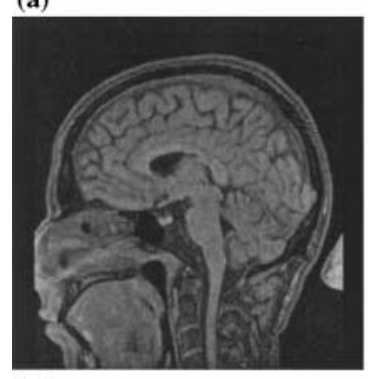

(c)

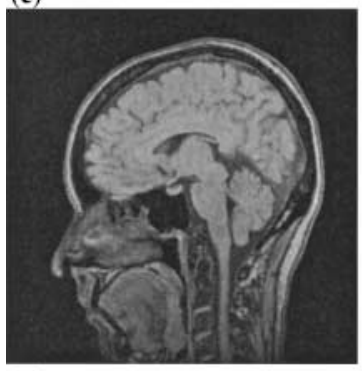

(e)

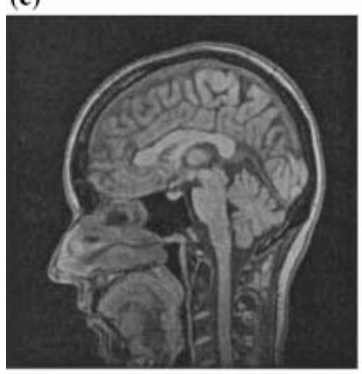

(g)

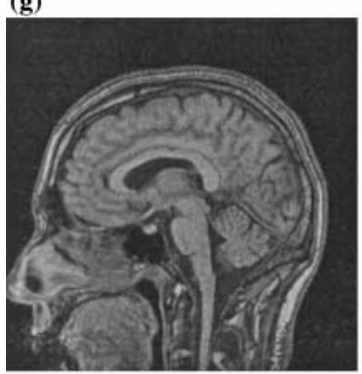

(i)

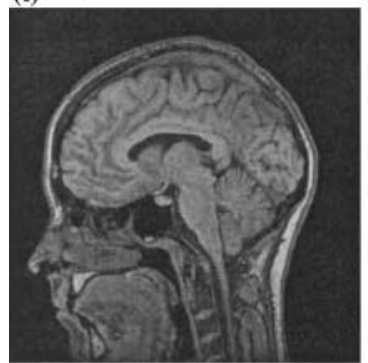

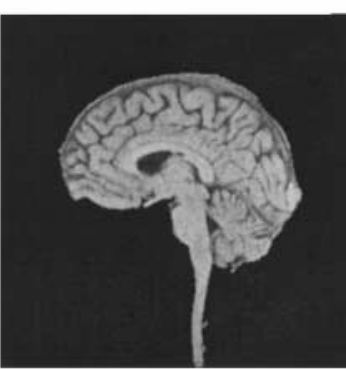
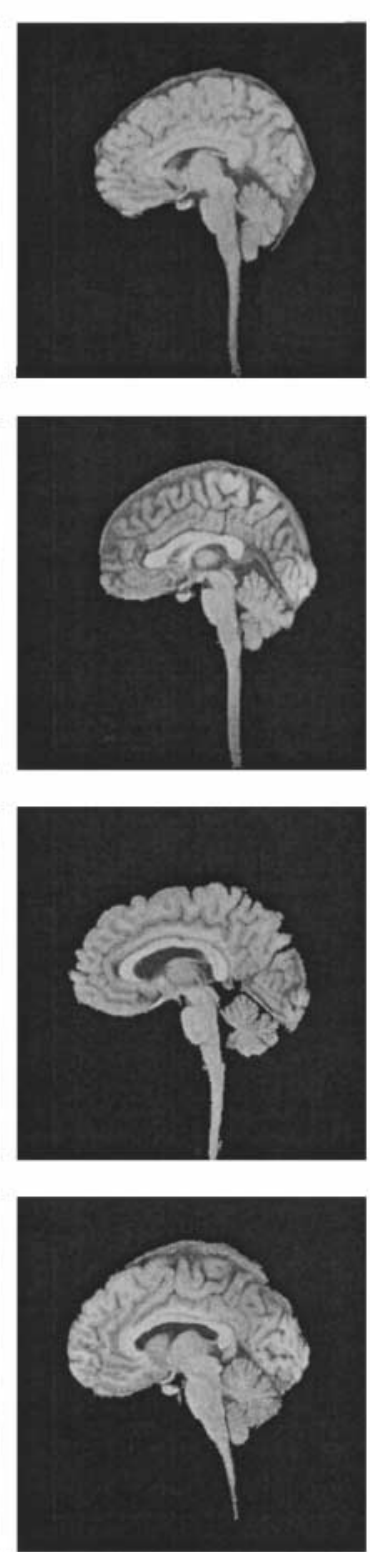

(b)
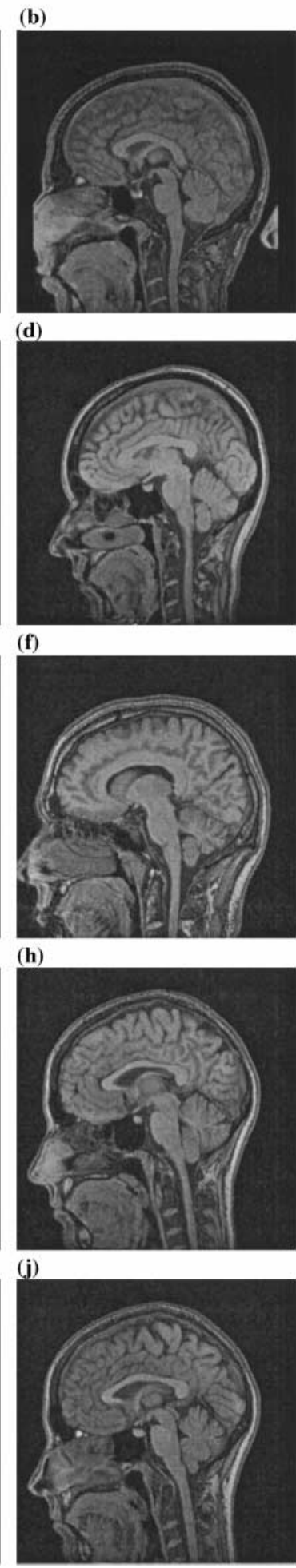
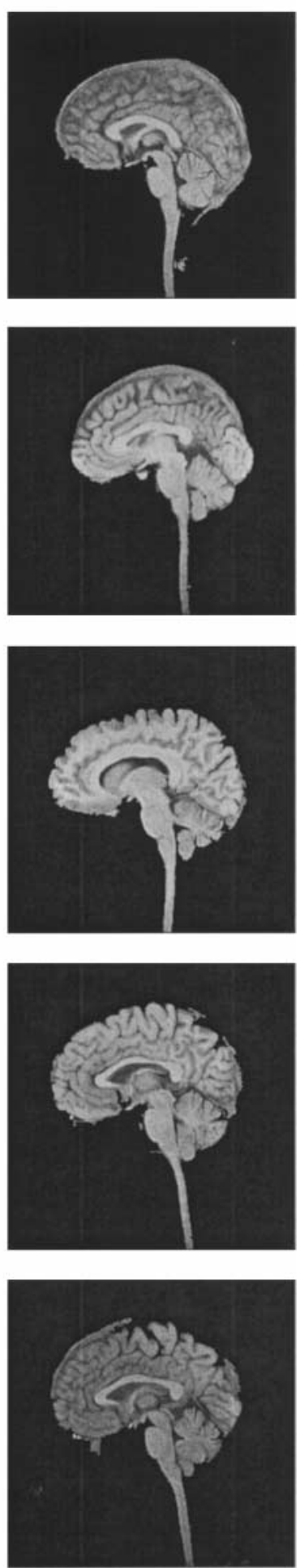

Fig. 30 . 


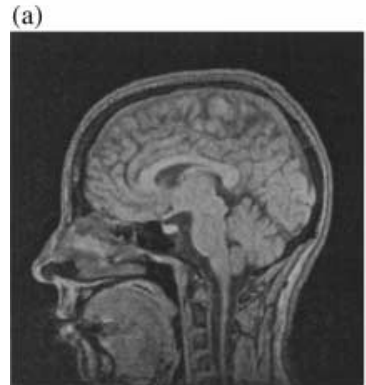

(c)

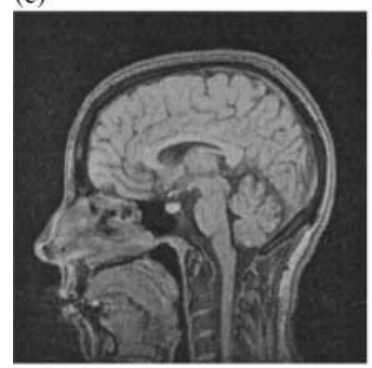

(e)

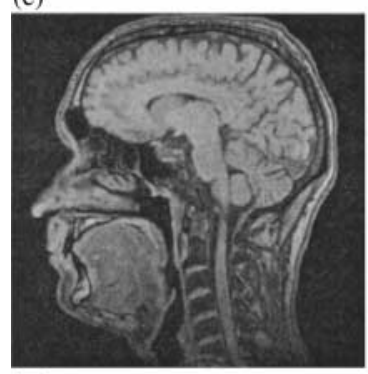

(g)

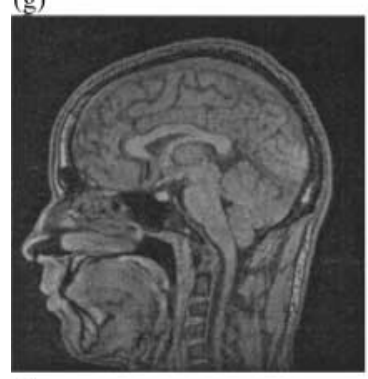

(i)

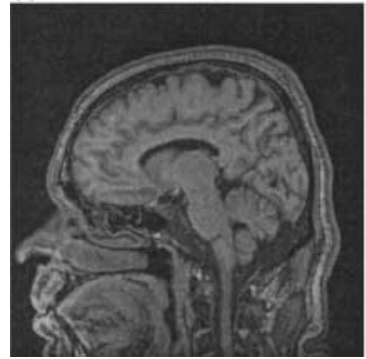

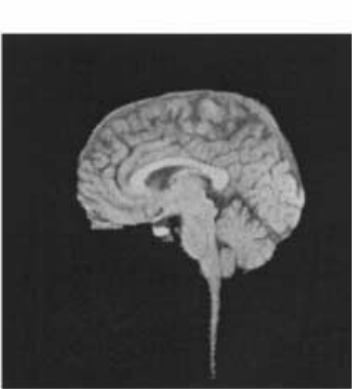
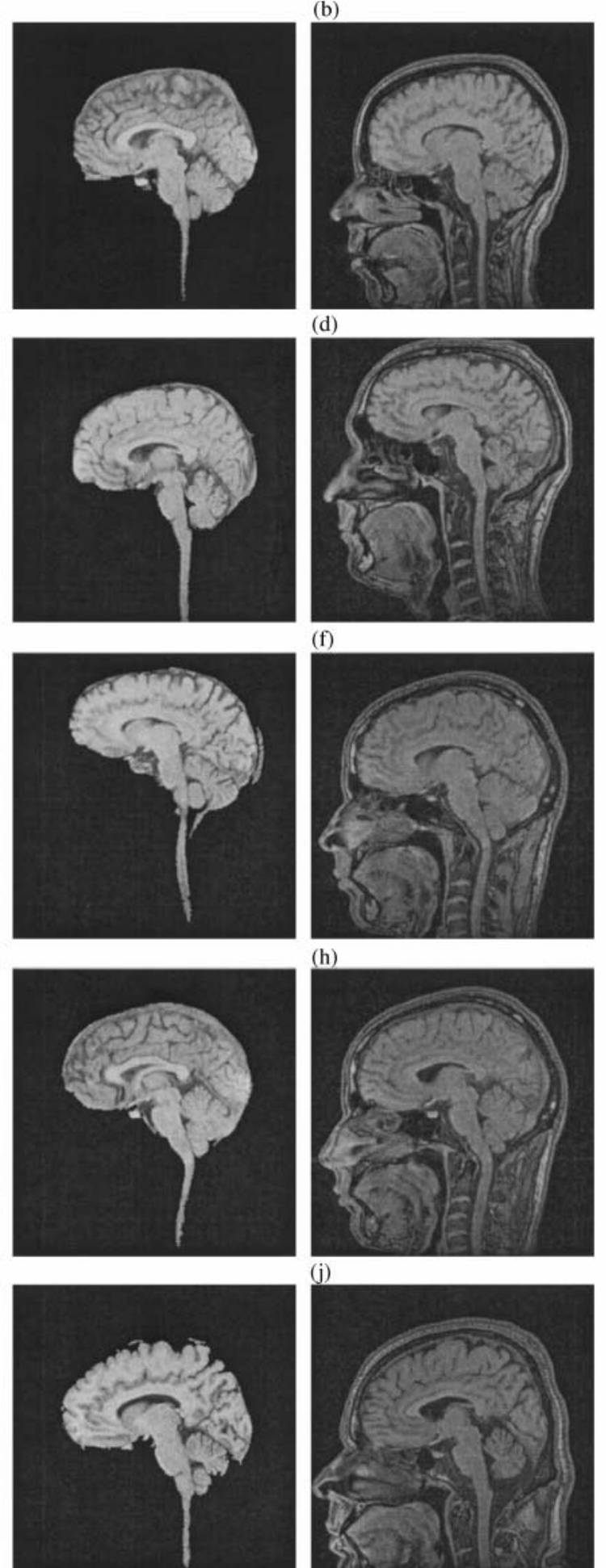

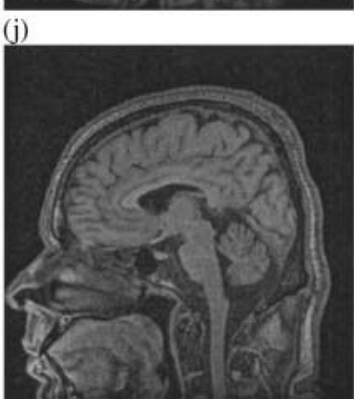

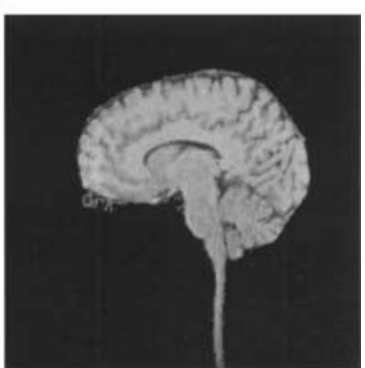
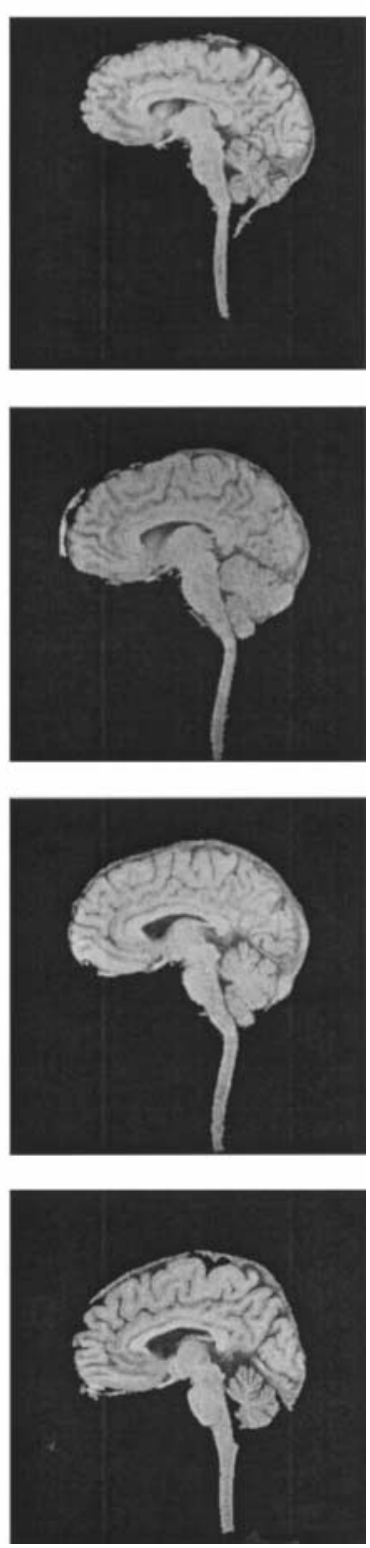

Fig. 31. 


\section{References}

[1] J.C. Bezdek, L.O. Hall, L.P. Clarke, Review of MR image segmentation techniques using pattern recognition, Medical Physics 20 (1993) 1033.

[2] R. Dekerck, C. Cornelis, M. Bister, Segmentation of medical images, Image and Vision Computing 11 (1993) 486.

[3] D.N. Kennedy, P.A. Filipek, J.V.S. Caviness, Anatomic segmentation and volumetric calculations in nuclear magnetic resonance imaging, IEEE Transactions on Medical Imaging. 8 (1989) 1.

[4] M. Joliot, B.M. Mazoyer, Three-dimensional segmentation and interpolation of magnetic resonance brain images, IEEE Transactions on Medical Imaging 12 (1993) 269.

[5] S.C. Amartur, D. Piraino, Y. Takefuji, Optimization neural networks for the segmentation of magnetic resonance images, IEEE Transactions on Medical Imaging 11 (1992) 215.

[6] M. Özkan, B.M. Dawant, R.J. Maciunas, Neural-network-based segmentation of multi-modal medical images: a comparative and prospective study, IEEE Transactions on Medical Imaging 12 (1993) 534.

[7] J. Kittler, J. Illingworth, Minimum error thresholding, Pattern Recognition 19 (1986) 41.

[8] S.U. Lee, S.Y. Chung, A comparative performance study of several global thresholding techniques for segmentation, Computer Vision Graphics Image Process. 52 (1990) 171.

[9] T. Pun, A new method for grey-level picture thresholding using the entropy of the histogram, Signal Processing $2(1980) 223$

[10] J.S. Weszka, A survey of threshold selection techniques, Computer Graphics and Image Processing 7 (1978) 259.

[11] T.N. Pappas, An adaptive clustering algorithms for image segmentation, IEEE Transactions on Signal Processing 40 (1992) 901.

[12] L.S. Davis, A survey of edge detection techniques, Computer Graphics Image Process 4 (1975) 248.

[13] J.F. Canny, A computational approach to edge detection, IEEE Transactions on Pattern Analysis and Machine Intelligence. 8 (1986) 679.

[14] R. Mohan, R. Nevatia, Perceptual organization for scene segmentation and description, IEEE Transactions on Pattern Analysis and Machine Intelligence 14 (1992) 616.

[15] C.-C. Chu, J.K. Aggarwal, The integration of image segmentation maps using region and edge information, IEEE Transactions on Pattern Analysis and Machine Intelligence 15 (1993) 1241.

[16] N.R. Pal, S.K. Pal, A review on image segmentation techniques, Pattern Recognition 26 (1993) 1277.

[17] K.S. Fu, J.K. Mui, A survey on image segmentation, Pattern Recognition 13 (1981) 3.

[18] R.M. Haralick, L.G. Shapiro, Survey, image segmentation techniques, Computer Vision Graphic Image Process 29 (1985) 100.

[19] G. Gerig, J. Martin, R. Kikinis, O. Kubler, M. Shenton, F.A. Jolesz, Unsupervised tissue-type segmentation of 3-D dual-echo MR head data, Image and Vision Computing 10 (1992) 349

[20] M. Bomans, K.-H. Höhne, U. Tiede, M. Riemer, 3-D segmentation of MR images of the head for 3-D display, IEEE Transactions on Medical Imaging 9 (1990) 177.

[21] C.Y. Lee, An algorithm for path connections and its applications, IRE Transactions on Electronic Computers 10 (1961) 346.

[22] F. Rubin, Path connection algorithm. IEEE Transactions on Computers (1974) 907.

[23] M. Unser, M. Eden, Multiresolution feature extraction and selection for texture segmentation, IEEE Transactions on PAMI 11 (1989) 717.

Chulhee Lee received the B.Sc. and M.Sc. degrees in electronics engineering from Seoul National University in 1984 and 1986, respectively, and the Ph.D. degree in electrical engineering from Purdue University, West Lafayette, IN, in 1992. From 1986 to 1987, he was a researcher in the Acoustic Laboratory at Technical University of Denmark (DTH). From 1993 to 1996, he worked with National Institutes of Health, Bethesda, MD. In 1996, he joined the faculty of the Department of Electronic Engineering, Yonsei University, Seoul. His research interests include image/signal processing, pattern recognition, and neural networks. Dr. Lee is a member of Tau Beta Pi, Eta Kappa Nu and KSEA.

Shin Huh was born in Seoul on February 22, 1976. He received the B.Sc. degree in electronics engineering from Yonsei University in 1998. He is currently working towards the M.Sc. degree in electronics engineering at Yonsei University. His current research interests include image segmentation and image/signal processing. 
Dr. Terence Ketter obtained his medical degree from the University of Toronto and had internship and residency training in psychiatry at the University of California at San Francisco. He had subsequent fellowship training in psychopharmacology and brain imaging research methods in the Biological Psychiatry Branch of the National Institute of Mental Health in Bethesda, MD. $\mathrm{He}$ is currently Associate Professor of Psychiatry and Behavioral Sciences and Chief of the Bipolar Disorders Clinic at the Stanford University School of Medicine in Stanford, California. Dr. Ketter's research interests include the use of brain imaging methods such as positron emission tomography (PET) and magnetic resonance imaging (MRI) and magnetic resonance spectroscopy (MRS) to better understand the neurobiology of mood (and especially bipolar) disorders. Dr. Ketter has also investigated the use of novel therapies such as new anticonvulsant drugs and combinations of medications in the treatment of mood disorders. His current work includes exploring the possibility of using brain imaging techniques to more effectively target treatments in mood disorders.

Michael Unser was born in Zug, Switzerland, on April 9, 1958. He received the M.Sc. (summa cum laude) and Ph.D. degrees in Electrical Engineering in 1981 and 1984, respectively, from the Swiss Federal Institute of Technology in Lausanne. From 1985 to 1997, he was with the Biomedical Engineering and Instrumentation Program, National Institutes of Health, Bethesda, where he had been heading the Image Processing Group. He is now Professor of Biomedical Image Processing at the Swiss Federal Institute of Technology in Lausanne, Switzerland. His research is centered around the numerical aspects of biomedical imaging. He has a strong interest in sampling theories, multiresolution algorithms, wavelets, and the use of splines for image processing. He is the author of over 70 published journal papers in these areas. Dr. Unser serves as an Associate Editor for the IEEE Signal Processing Letters, and is a member of the Image and Multidimensional Signal Processing Committee of the IEEE Signal Processing Society. $\mathrm{He}$ is also on the editorial boards of Signal Processing, the Journal of Visual Communication and Image Representation, Pattern Recognition, and was a former Associate Editor (1992-1995) for the IEEE Transactions on Image Processing. He coorganized the 1994 IEEE-EMBS workshop on wavelets in medicine and biology, and serves as conference chair for SPIE's Wavelet applications in signal and image processing, which has been held annually since 1993. He received the Dommer prize for excellence from the Swiss Federal Institute of Technology in 1981, the research prize of the Brown-Boveri Corporation, Switzerland, for his thesis in 1984 and the IEEE Signal Processing Society's 1995 Best Paper Award (IMDSP technical area) for a Transactions paper with A. Aldroubi and M. Eden on B-spline signal processing. 